\title{
Boundary conditions and localization on AdS. Part II. General analysis
}

\author{
Justin R. David, ${ }^{a}$ Edi Gava, ${ }^{b, d}$ Rajesh Kumar Gupta ${ }^{c}$ and Kumar Narain ${ }^{d}$ \\ ${ }^{a}$ Centre for High Energy Physics, Indian Institute of Science, \\ C.V. Raman Avenue, Bangalore 560012, India \\ ${ }^{b}$ INFN, Sezione di Trieste, \\ Strada Costiera 11, Trieste, Italy \\ ${ }^{c}$ Department of Mathematics, King's College London, \\ The Strand, London WC2R 2LS, U.K. \\ ${ }^{d}$ ICTP, Strada Costiera 11, Trieste 34151, Italy \\ E-mail: justin@cts.iisc.ernet.in, gava@ictp.it, \\ rajesh.gupta@kcl.ac.uk, narain@ictp.it
}

ABSTRACT: We develop the method of Green's function to evaluate the one loop determinants that arise in localization of supersymmetric field theories on AdS spaces. The theories we study have at least $\mathcal{N}=2$ supersymmetry and normalisable boundary conditions are consistent with supersymmetry. We then show that under general assumptions the variation of the one loop determinant with respect to the localizing background reduces to a total derivative. Therefore it receives contributions only from the origin of AdS and from asymptotic infinity. From expanding both the Greens function and the quadratic operators at the origin of AdS and asymptotic infinity, we show that the variation of the one loop determinant is proportional to an integer. Furthermore, we show that this integer is an index of a first order differential operator. We demonstrate that these assumptions are valid for Chern-Simons theories coupled to chiral multiplets on $A d S_{2} \times S^{1}$. Finally we use our results to show that $\mathrm{U}\left(N_{c}\right)$ Chern-Simons theory at level $k$ coupled to $N_{f}$ chiral multiplets and $N_{f}$ anti-chiral multiplets in the fundamental obeys level-rank duality on $A d S_{2} \times S^{1}$.

KEYwords: Extended Supersymmetry, Field Theories in Lower Dimensions, Supersymmetric Gauge Theory

ArXiv EPRINT: 1906.02722 


\section{Contents}

1 Introduction $\quad 1$

2 A general proof $\quad 5$

2.1 Green's function 8

\begin{tabular}{ll}
2.2 & Variation of one loop determinant \\
\hline & 12
\end{tabular}

$\begin{array}{ll}2.3 \text { Calculation of the boundary terms } & 14\end{array}$

$\begin{array}{lll}2.4 & \text { Connection to index of the operator } C & 18\end{array}$

3 Chern-Simons theory on $A d S_{2} \times S^{1}$ : Greens function approach $\quad 19$

3.1 Q-exact deformation and gauge fixing 20

$\begin{array}{ll}3.2 & \text { Boundary conditions } \\ 3.3 & 22\end{array}$

3.3 Equations of motions and the Greens function 23

$\begin{array}{ll}3.4 & \text { Boundary terms }\end{array}$

3.5 Evaluating boundary terms 26

3.6 Variation of the one loop partition function 33

4 Level-rank duality on $A d S_{2} \times S^{1}$

$\begin{array}{lll}5 & \text { Conclusions } & 37\end{array}$

$\begin{array}{ll}\text { A Supersymmetry of the vector multiplet } & 38\end{array}$

B Equations of motions $\quad 39$

C On gauge fixing conditions $\quad 42$

\section{Introduction}

Supersymmetric localization on compact spaces and its applications has been studied extensively recently, see [1] for a recent review. This area began with the work of Witten [2] and was developed in the works of [3-5] to enable the evaluation of observables in supersymmetric quantum field theories. The exact computation of supersymmetric partition functions and Wilson lines served as highly non-trivial checks of AdS/CFT [6-8]. Field theories defined on a compact space serve as standard examples for applying the method of localization. This is because the method relies on identifying a fermionic symmetry $Q$. The Lagrangian including the localizing term is symmetric under $Q$ only upto boundary terms and restricting the space to be compact ensures that these boundary terms do not arise. 
The systematic extension of the method of supersymmetric localization is an important problem. Non-compact spaces which form the canonical examples to study localization are of the form $A d S_{n} \times S^{m}$. This is mainly due to the variety of applications of supersymmetric theories on such spaces. For example, localization of $\mathcal{N}=2$ gravity on $A d S_{2} \times S^{2}$ is important for obtaining the exact entropy of BPS black holes in these theories [9-14]. Similarly the exact evaluation of the supersymmetric partition function of $\mathcal{N}=8$ supergravity on $A d S_{4}$ serves as an important check of the holographic duality with ABJM theory $[15,16]$.

As demonstrated in [17], when the method of supersymmetric localization is applied to non compact spaces one needs to carefully examine the boundary conditions implemented on the fields. ${ }^{1}$ The boundary conditions of both the bosonic and fermionic fields must be chosen so that they are consistent with the superysmmetric transformations. They also must be chosen so that boundary terms that arise under the action of $Q$ on both the original action as well as the localizing term vanish. Furthermore, the path integral must be well defined under these boundary conditions. Normalizable boundary conditions on all fields ensure that the boundary terms at asymptotic infinity vanish as well as the path integral is well defined. However normalizable boundary conditions may not always be compatible with supersymmetry.

In [18], the method of Greens function was introduced to evaluate one loop determinants that arise in localization. This was done for the $\mathcal{N}=2$ chiral multiplet on $A d S_{2} \times S^{1}$. The method involved studying the variation of the one loop determinant under a parameter $\alpha,{ }^{2}$ that parametrises the localizing background and then integrating with respect to $\alpha$. It was shown that whenever normalizable boundary conditions also are consistent with supersymmetric transformations, the variation of the one loop determinant reduces to a total derivative and one only needs to evaluate the boundary contributions from the origin of $A d S_{2}$ and the asymptotic infinity. Furthermore, it was demonstrated that the final result for the one loop determinant agrees with the index whenever the boundary conditions are normalizable and supersymmetric.

In this paper we develop the Green's function method further. A brief outline of the Greens function method is the following. Let $\mathcal{D}_{B}(\alpha)$ be the bosonic operator and $\mathcal{D}_{F}(\alpha)$ be the fermionic operator that occurs in the evaluation of the one loop determinants. They depend on the classical localising background through the parameter $\alpha$. Then the variation of the one loop determinants with respect to $\alpha$ is given by

$$
\frac{\delta}{\delta \alpha} \ln Z_{\text {1-loop }}(\alpha)=\operatorname{Tr}\left[G_{F} \frac{\delta}{\delta \alpha} \mathcal{D}_{F}(\alpha)\right]-\frac{1}{2} \operatorname{Tr}\left[G_{B} \frac{\delta}{\delta \alpha} \mathcal{D}_{B}(\alpha)\right]
$$

Here $G_{B}, G_{F}$ are the bosonic and fermionic Greens function corresponding to the operator $\mathcal{D}_{B}$ and $\mathcal{D}_{F}$ respectively.

We show that under some general assumptions which hold for theories with at least $\mathcal{N}=2$ supersymmetry on spaces of the form $A d S_{n} \times S^{m}$ the variation of the one loop determinant with respect to $\alpha$ which parametrises the localising backround always reduces

\footnotetext{
${ }^{1}$ See also [19] where the partition function for a chiral multiplet on the twisted background of $\mathrm{AdS}_{2} \times$ $\mathrm{S}^{1}$ with flavor fugacity turned on was computed using the localization technique.

${ }^{2} \alpha$ parametrises the vector multiplet background.
} 
to a total derivative. This reduction to a total derivative holds whenever supersymmetric boundary conditions are compatible with normalisable boundary conditions. The general assumptions that we make relate to the properties of the second order operators, $\mathcal{D}_{B}$ and $\mathcal{D}_{F}$, that arise in these theories in the evaluation of the one loop determinants. These assumptions enable the evaluation of the variation given in (1.1). Then integrating with respect to $\alpha$ we can obtain the one loop determinant. In this paper we demonstrate that these properties hold for both the vector multiplet as well as the chiral multiplet on $A d S_{2} \times S^{1}$. We have also verified that it continues to hold for the vector as well as the hypermultiplet on $A d S_{2} \times S^{2}$ [20]. We suspect that the general assumptions are properties that hold whenever the actions have at least $\mathcal{N}=2$ supersymmetry but at present we do not have a proof.

Here we state these assumptions in a qualitative form. In the next section we make these quantitative. These assumptions are made on the second order differential operators that appear after one reduces the operators $\mathcal{D}_{B}, \mathcal{D}_{F}$ to only the radial equation parametrising the AdS direction. This reduction is made by expanding all the fields in an appropriate basis. For example, it is the Fourier basis corresponding to the two $S^{1}$ 's for the case of $A d S_{2} \times S^{1}$

1. The matrix second order operator corresponding to the one loop bosonic determinant reduces to a certain block diagonal form in a special gauge. The operators are Hermitian and non-degenerate and have regular singularities at the origin of AdS and the boundary. This last assumption enables a Forbenius series expansion of the solutions at these points.

2. The matrix second order operator corresponding to the one loop fermionic determinants also reduce to a certain block form. All the second derivatives occur only with terms involving the ghosts. The operator is Hermitian. It is only certain components of the block form that contain the dependence on $\alpha$ which parametrises the background.

3. The bosonic operator and the fermionic operators are related to each other by factors of $Q^{2}$. This follows from supersymmetry. Therefore the fermionic solutions can be found in terms of the bosonic ones.

4. The Greens function for the bosonic operator exists and this in turn implies the Greens function for the fermionic operator can be constructed from that of the bosonic Greens function.

Using these assumptions it can be shown that the variation in (1.1) reduces to a total derivative. Therefore, the behaviour of the Greens functions as well as the second order operators at the origin of AdS and at infinity determine the variation (1.1). The result for the variation is given in equation (2.31). Then finally using assumptions of the behaviour of certain components of the fermionic matrix operator at these points, the variation can be evaluated. 
Our main result is that we show that the variation of the one loop determinant given in (1.1) is an integer times the variation of $\frac{1}{2} \ln \left(Q^{2}\right)$. The integer is determined by the difference between the number of allowed solutions to a first order differential equation that occurs from the fermionic operator at the origin and at asymptotic infinity of the AdS. The result is given in equation (2.47). Thus, the final result for the one loop determinant resembles an index of an operator. We then identify this operator and show that the one loop determinant is expressed in terms of index of this operator.

As we mentioned earlier we verify that these assumptions hold for the case of the $\mathcal{N}=2$ vector as well as the chiral multiplet on $A d S_{2} \times S^{1}$. We also show that normalisable boundary conditions imply supersymmetric boundary conditions for the vector multiplet provided $L^{2}>\frac{3}{4}$. Here $L$ is the ratio of the radius of $A d S_{2}$ to $S^{1}$. For the chiral multiplet of R-charge $\Delta$ the conditions that ensure normalisable boundary conditions are also supersymmetric is that there should be no integer $n$ in the interval $\left(\frac{\Delta-1}{2 L}, \frac{\Delta}{2 L}\right)$ was obtained in $[18]$.

We apply our results to $\mathcal{N}=2$ Chern Simons with $N_{f}$ chiral multiplets in the fundamental and $N_{f}$ anti-chiral multiplets in the fundamental and show that the partition function of the theory with gauge group $\mathrm{U}\left(N_{c}\right)$ at level $k$ is identical to the theory with the gauge group $\mathrm{U}\left(|k|+N_{f}-N_{c}\right)$ at level $-k$ and with the same matter content. That is level-rank duality continues to hold when the theory is placed on $A d S_{2} \times S^{1}$.

It is important to mention that our gauge fixing condition is a generalisation of the covariant gauge condition which is given by

$$
\cosh ^{2} r \nabla^{\hat{\mu}}\left(\frac{1}{\cosh ^{2} r} a_{\hat{\mu}}\right)+\partial_{t} a_{t}=0 .
$$

This gauge condition was first used in [17]. Here $r$ is the radial coordinate in $A d S_{2}, \hat{\mu}$ refers to the two coordinates on $A d S_{2}$ and $t$ refers to the coordinate on $S^{1}$. This gauge choice ensures that the operators that occur the operators that occur in the analysis of the Greens function of the bosons is block diagonal. We have seen that the results are independent of gauge choice. We show in appendix $\mathrm{C}$ that for the bosonic $\mathrm{U}(1)$ Chern-Simons theory, the partition function evaluated in a one parameter set of gauge conditions that interpolate between the covariant gauge and the condition in (1.2) remains the same.

This paper is organised as follows. In section 2 we present the details of the assumptions made on the properties of the quadratic operators that appear in localization of at least $\mathcal{N}=2$ theories on AdS spaces. In section 2.3 we make further assumptions on the behaviour of the terms in the fermionic kinetic term at the boundary of AdS and at the origin. We then present our proof that under these assumptions the variation of the one loop determinant is an integer times the variation of $\frac{1}{2} \ln \left(Q^{2}\right)$ is given in section 2.3. In section 2.4, we show that this integer is the index of a first order matrix differential operator appearing in the fermionic kinetic term. In section 3 we introduce $\mathcal{N}=2$ Chern Simons theory on $A d S_{2} \times S^{1}$, the localizing term as well as the gauge fixing condition. We also determine the behaviour of all fields at asymptotic infinity of $A d S_{2}$ so that they are all normalisable. In section 3.3, we demonstrate that the general assumptions made on the properties of the second operators that occur in evaluating one loop determinants in section hold for the case 
of $\mathcal{N}=2$ Chern-Simons theory on $A d S_{2} \times S^{1}$. We also derive the conditions under which normalizable boundary conditions are consistent with supersymmetry. Finally we obtain the variation of the one loop determinant and demonstrate that it is an integer times the variation of $\frac{1}{2} \ln \left(Q^{2}\right)$. We show that the result agrees with that obtained in [17]. In section 4 we apply our analysis to evaluate the supersymmetric partition function of $\mathrm{U}\left(N_{c}\right)$ ChernSimons theory on $A d S_{2} \times S^{1}$ coupled with $N_{f}$ chiral multiplets in the fundamental and an equal number of chiral multiplets in the anti-fundamental. From the expression of the partition function we demonstrate this theory obeys level-rank duality. Section 5 contains our conclusions. Appendix A and B provide the details of the supersymmetic variations as well as the equations of motion of all the fields about the localization background. Appendix C contains the evaluation of the partition function of $\mathrm{U}(1)$ Chern-Simons theory in a one parameter set of gauge conditions which interpolate between the covariant gauge and the gauge in (1.2).

\section{A general proof}

In this section, we will present a general discussion about the one loop computations in supersymmetric localization on a general manifold for vector and matter multiplets. Our discussion will be based on the Green's function method which was used in [18] to compute the path integral of chiral multiplet on $\mathrm{AdS}_{2} \times \mathrm{S}^{1}$. In the computation of path integral using the supersymmetric localization technique, we need to compute the one loop determinant of the operators about the localization background. In the Green's function approach, developed in [18], we computed the variation of the one loop determinant instead i.e.

$$
\frac{\delta}{\delta \alpha} \ln Z_{1 \text {-loop }}(\alpha)=\operatorname{Tr}^{\prime}\left[G_{F} \frac{\delta}{\delta \alpha} \mathcal{D}_{F}(\alpha)\right]-\frac{1}{2} \operatorname{Tr}^{\prime}\left[G_{B} \frac{\delta}{\delta \alpha} \mathcal{D}_{B}(\alpha)\right]
$$

where $\mathcal{D}_{F}(\alpha)$ and $\mathcal{D}_{B}(\alpha)$ are fermionic and bosonic kinetic operator, respectively and $G_{F}$ and $G_{B}$ are their Green's functions. Also, $\alpha$ is some parameter which enters in both bosonic and fermionic differential operator and the "Tr" in (2.1) is the space-time as well as matrix trace over non zero modes. Typically, we choose this parameter to the one which parametrises the localization background. The one loop determinant, up to a constant in $\alpha$, is then obtained by integrating the right hand side of (2.1) with respect to $\alpha$.

The choice of the parameter $\alpha$ is arbitrary as it was shown in [18], the final result of the one loop determinant is independent of the choice of the parameter with respect to which we decide to vary the one loop determinant. Thus in this method, we need compute the Green's function of the differential operator which appears in the one loop computations. One of the remarkable simplifications occur in this approach is that when the boundary conditions of the fields are consistent with supersymmetry, the variation (2.1) is a total derivative and contributions to one loop determinant comes from the boundary behaviour of the solutions of equations of motion of all the fields in the chiral multiplet. We find that this is quite generic feature of the supersymmetric localization and independent of the multiplet and spaces i.e. if the boundary conditions of the fields are consistent with supersymmetry, the variation is always a total derivative. 
Our method presented below is quite generic and, in particular, very useful for the localization computation in non compact spaces such as AdS space which also involve imposing a boundary conditions. We start with stating the notation and the set up.

\section{Set up.}

1. In the vector multiplet fields, after integrating out the auxiliary fields as well as $b$ (BRST partner of the ghost $\widetilde{c}$ ) ghost we are left with the vector field, scalar fields, ghost field $c$ and fermions. We denote the bosonic fields by $X_{0}$ and $\sigma$, where $\sigma$ is the scalar field which parametrises the localization manifold. The bosonic field $X_{0}$ is a $(k+1)$ component column vector. In the case of $A d S_{2} \times S^{1}$, we have $k+1=3, X_{0}$ consists of the gauge field $a_{\hat{\mu}}$, $a_{t}$. Since the method always requires a scalar which takes a non zero value on the localization manifold, the method is suitable for theories with at least $\mathcal{N}=2$ supersymmetry.

2. The fermionic fields are grouped as $Q X_{0}$ and $\left(c, X_{1}^{\prime}\right)$. The fermionic field $Q X_{0}$ and $X_{1}^{\prime}$ are $(k+1)$ and $k$ component column vector, respectively.

3. In the matter multiplet fields, after integrating out the auxiliary fields we are left with scalar fields which we denote by $X_{0}$ and the fermionic fields are decomposed as $Q X_{0}$ and $X_{1}$. We assume that the scalar fields in the matter multiplet do not acquire non zero value on the localization manifold.

With this set up, our method of localization computations will be based on the following assumptions:

\section{Assumptions.}

1. Fields are functions of a non periodic coordinate $r$. In particular, it is assumed that we have done Kaluza Klein reduction in the rest of the coordinate and the Lagrangian for each KK mode is a function in one variable $r$. We will take the range of $r$ to be from 0 to $\infty$ for convenience (precise interval is not important for most of the presentation).

2. For the vector multiplet calculations, we need to add gauge fixing functional $\mathcal{G}(A)$ in the path integral. We assume that the gauge fixing condition $\mathcal{G}(A)$ is such that after eliminating auxiliaries and $b$, the bosonic equation for $\sigma$ decouples from the rest of the bosonic fields $X_{0} \cdot{ }^{3}$ This choice is not necessary but it will simplify some of the calculations. The bosonic equations can, therefore, be written as the matrix operator

$$
\left(\begin{array}{cc}
A_{1}^{b}(r) & 0 \\
0 & A_{2}^{b}(r)
\end{array}\right)\left(\begin{array}{c}
X_{0} \\
\sigma
\end{array}\right) \equiv M_{b}\left(\begin{array}{c}
X_{0} \\
\sigma
\end{array}\right) .
$$

For the matter multiplet case there is no second block corresponding to $\sigma$.

\footnotetext{
${ }^{3}$ For the vector multiplet case we add the following gauge fixing term in the $Q V$ action: $Q \operatorname{tr}(\tilde{c}(\mathcal{G}+\xi b))$ where $\xi$ is a parameter. It turns out that in order to decouple the equations of motion of $\sigma$ field from rest of the bosonic fields, one needs to add a $Q$-exact term to the localizing action of the form $Q(\operatorname{tr}(\alpha[c, \mathcal{G}]))$, where $\alpha$ is some constant (in general it is related to localization background), which can also be thought of as redefining $\tilde{c} \rightarrow \tilde{c}+[\alpha, c]$.
} 
3. We assume that $M_{b}$ is hermitian second order matrix differential operator:

$$
M_{b}(r)=M_{b}^{(2)}(r) \frac{d^{2}}{d r^{2}}+M_{b}^{(1)}(r) \frac{d}{d r}+M_{b}^{(0)}(r)
$$

where $M_{b}^{(2)}(r), M_{b}^{(0)}(r)$ and $M_{b}^{(0)}(r)$ are $(k+2) \times(k+2)$ matrices and $M_{b}^{(2)}(r)$ is nondegenerate. It implies that $A_{1}^{b}(r)$ and $A_{2}^{b}(r)$ are hermitian and second order matrix differential operators and the coefficient of $\frac{d^{2}}{d r^{2}}$ is non-degenerate for all $r \in(0, \infty)$. At $r=0$ and $u=e^{-r}=0$ (i.e. the two boundaries of the one-dimension problem) and the operators $A_{1}^{b}$ and $A_{2}^{b}$ have regular singularities.

4. For the fermionic fields the equations are:

$$
\left(\begin{array}{ccc}
A_{11}(r) & A_{12}(r) & B(r) \\
A_{21}(r) & A_{22}(r) & 0 \\
C(r) & 0 & D(r)
\end{array}\right)\left(\begin{array}{c}
Q X_{0} \\
c \\
X_{1}^{\prime}
\end{array}\right) \equiv M_{f}(r)\left(\begin{array}{c}
Q X_{0} \\
c \\
X_{1}^{\prime}
\end{array}\right) .
$$

Here, generically, $A_{11}(r), A_{12}(r), A_{21}(r)$ and $A_{22}(r)$ are $(k+1) \times(k+1),(k+1) \times 1,1 \times$ $(k+1)$ and $1 \times 1$ matrix differential operators, respectively. Similarly, $B(r), C(r)$ and $D(r)$ are $(k+1) \times k, k \times(k+1)$ and $k \times k$ matrix differential operators, respectively.

5. $M_{f}(r)$ is assumed to be Hermitian. In particular, this means that $A_{11}(r), A_{22}(r)$ and $D(r)$ are Hermitian while $A(r)_{21}^{\dagger}=A_{12}(r)$ and $B(r)=C(r)^{\dagger}$.

6. $D(r)$ is purely algebraic and is invertible $k \times k$ matrix. $A_{11}(r), B(r)$ and $C(r)$ involve only first order differential operators. The only two derivative term in the localizing action are the ones that involve ghost $c$. What this means is that $A_{21}(r), A_{12}(r)$ and $A_{22}(r)$ involve second order differential operators.

7. Requiring that the action is supersymmetric implies that one can obtain the equations of motions for fermionic fields from those of the bosonic fields upto a factor of $Q^{2}$. This implies that there exist a matrix first order differential operator $E$ and its adjoint $E^{\dagger}$ such that

$$
\hat{M}(r) \equiv E(r)^{\dagger} M_{f}(r) E(r)=\left(\begin{array}{ccc}
\gamma_{1} A_{1}^{b}(r) & 0 & 0 \\
0 & \gamma_{2} A_{2}^{b}(r) & \\
0 & 0 & D(r)
\end{array}\right) .
$$

It is not very hard to find $E(r)$ which does the above and is given by

$$
E(r)=\left(\begin{array}{ccc}
1 & 0 & 0 \\
K & f(r) & 0 \\
-D(r)^{-1} C(r) & 0 & 1
\end{array}\right)
$$


and for this choice of $E(r)$, the constants are $\gamma_{1}=\frac{1}{Q^{2}}$ and $\gamma_{2}=Q^{2}$. Here $K$ is a $(k+1)$-component row vector and $f(r)$ is a scalar function which is independent of the parameter $\alpha$. More explicitly, the relations are ${ }^{4}$

$$
\begin{aligned}
A_{12}(r) & =-K^{\dagger} A_{22}(r), & A_{21}(r) & =-A_{22}(r) K \\
A_{11}(r)-K^{\dagger} A_{22}(r) K-B(r) D(r)^{-1} C(r) & =\gamma_{1} A_{1}^{b}(r), & f(r)^{\dagger} A_{22}(r) f(r) & =\gamma_{2} A_{2}^{b}(r) .
\end{aligned}
$$

8. The Greens fn for $A_{1}^{b}$ exists. This means that $A_{1}^{b}$ has no zero modes. The differential operator $A_{2}^{b}$ can have zero modes. Typically, these correspond to the variation of the saddle point, which happens only for modes that are constant along space orthogonal to $A d S_{2}$ and for which we already have collective coordinate integration. This case will be discussed separately.

\subsection{Green's function}

In this section, we will construct the Green's function for both the fermionic and bosonic kinetic operators and discuss the relation between the two. We will find that the fermionic Green's function can always be constructed from the bosonic Green's function provided their boundary conditions agree with supersymmetry.

We start with the bosonic Green's function. The bosonic Green's function satisfies the equation

$$
M_{b}(r) G_{b}\left(r, r^{\prime}\right)=\delta\left(r, r^{\prime}\right) I_{k+2} .
$$

Here $I_{k+2}$ is $(k+2)$-dimensional identity matrix. In general, the differential operator $M_{b}(r)$ could have zero modes. Since, in the path integral we integrate over only non zero modes, therefore, we are interested in computing only the Green's function for the non zero modes.

Let the solution for the Green's function equation for $r<r^{\prime}$ be

$$
G_{b}^{<}\left(r, r^{\prime}\right)=\left(\begin{array}{cc}
G_{1}\left(r, r^{\prime}\right) & 0 \\
0 & G_{2}\left(r, r^{\prime}\right)
\end{array}\right)
$$

and for $r>r^{\prime}$ be

$$
G_{b}^{>}\left(r, r^{\prime}\right)=\left(\begin{array}{cc}
G_{1}^{\prime}\left(r, r^{\prime}\right) & 0 \\
0 & G_{2}^{\prime}\left(r, r^{\prime}\right)
\end{array}\right)
$$

Furthermore, $G_{b}^{<}\left(r, r^{\prime}\right)$ is smooth at $r=0$ and satisfy the allowed boundary conditions at $r^{\prime}=\infty$ while $G_{b}^{>}\left(r, r^{\prime}\right)$ is smooth at $r^{\prime}=0$ and satisfy the allowed boundary conditions at $r=\infty$. It is important to note that these boundary conditions on the Green's function are exactly the same boundary condition which impose on the bosonic fields.

\footnotetext{
${ }^{4}$ The similarity transformations $(2.5)$ are obtained by following supersymmetry which implies that the equations of motion for $X_{0}$ and $Q X_{0}$ are identical upto a factor of $Q^{2}$. Similarly, the equation of motion for the ghost field $c$ is also related to $\sigma$. This relations follows because $Q c=f(r) \sigma+k \cdot X_{0}$ where $k$ is some vector which is usually related to the killing vector. If we define the field $c^{\prime}$ as $c^{\prime}=f(r)^{-1}\left(c-\frac{1}{Q^{2}} k \cdot Q X_{0}\right)$, then we see that supersymmetry implies the equation of motion for $c^{\prime}$ is identical to that of $\sigma$ upto a factor of $Q^{2}$.
} 
Since, $M_{b}(r)$ is a 2 nd order differential operator, these Green's function also satisfy the continuity/discontinuity relations:

$$
\begin{aligned}
\lim _{r^{\prime} \rightarrow r}\left(G_{b}^{>}\left(r, r^{\prime}\right)-G_{b}^{<}\left(r, r^{\prime}\right)\right) & =0, \\
\lim _{r^{\prime} \rightarrow r} \partial_{r}\left(G_{b}^{>}\left(r, r^{\prime}\right)-G_{b}^{<}\left(r, r^{\prime}\right)\right) & =\left(M_{b}^{(2)}(r)\right)^{-1} .
\end{aligned}
$$

Next, we will determine the fermionic Green's function. The Green's function equation for fermionic operator is

$$
M_{f}(r) G_{f}\left(r, r^{\prime}\right)=\delta\left(r, r^{\prime}\right) I_{2 k+2} .
$$

Now, following our assumption (6), the fermionic Green's function can be obtained from the bosonic Green's function i.e. for $r<r^{\prime}$, the fermionic Green's function is

$$
G_{f}^{<}\left(r, r^{\prime}\right) \equiv E(r) \hat{G}^{<}\left(r, r^{\prime}\right) E^{\dagger}\left(r^{\prime}\right)=E(r)\left(\begin{array}{ccc}
\frac{1}{\gamma_{1}} \widetilde{G}_{1}\left(r, r^{\prime}\right) & 0 & 0 \\
0 & \frac{1}{\gamma_{2}} \widetilde{G}_{2}\left(r, r^{\prime}\right) & 0 \\
0 & 0 & 0
\end{array}\right) E^{\dagger}\left(r^{\prime}\right)
$$

and for $r>r^{\prime}$

$$
G_{f}^{>}\left(r, r^{\prime}\right) \equiv E(r) \hat{G}^{>}\left(r, r^{\prime}\right) E^{\dagger}\left(r^{\prime}\right)=E(r)\left(\begin{array}{ccc}
\frac{1}{\gamma_{1}} \widetilde{G}_{1}^{\prime}\left(r, r^{\prime}\right) & 0 & 0 \\
0 & \frac{1}{\gamma_{2}} \widetilde{G}_{2}^{\prime}\left(r, r^{\prime}\right) & 0 \\
0 & 0 & 0
\end{array}\right) E^{\dagger}\left(r^{\prime}\right) .
$$

Here, it is worth to mention couple of points. Firstly, the bosonic Green's function $\widetilde{G}_{1,2}$ and $\widetilde{G}_{1,2}^{\prime}$ are such that the fermionic Green's function $G_{f}^{<}\left(r, r^{\prime}\right)$ and $G_{f}^{>}\left(r, r^{\prime}\right)$ satisfy the required boundary conditions as a function of both the argument $r$ and $r^{\prime}$. Therefore, in general $\widetilde{G}_{1,2}$ and $\widetilde{G}_{1,2}^{\prime}$ are different than $G_{1,2}$ and $G_{1,2}^{\prime}$, respectively. In particular, it satisfies

$$
\hat{M}(r) \hat{G}\left(r, r^{\prime}\right)=\delta\left(r, r^{\prime}\right)\left(\begin{array}{ccc}
I_{k+1} & 0 & 0 \\
0 & 1 & 0 \\
0 & 0 & 0
\end{array}\right) .
$$

Now, when the boundary conditions are consistent with supersymmetry then one can see that given an admissible bosonic solution one can construct an admissible fermionic solution and vice versa. Thus, for the supersymmetric boundary conditions we have $G_{1,2}\left(r, r^{\prime}\right)=$ $\widetilde{G}_{1,2}\left(r, r^{\prime}\right)$ and $G_{1,2}^{\prime}\left(r, r^{\prime}\right)=\widetilde{G}_{1,2}^{\prime}\left(r, r^{\prime}\right)$. The argument for this is as follows.

Let us suppose that $s$, which is a $(k+2)$-vector, is a solution of the bosonic equation $M_{b} s=0$. Now, consider the $(2 k+2)$ dimensional vector $s_{f}=E \hat{s}$ where $\hat{s}=\left(\begin{array}{l}s \\ 0\end{array}\right)$, where $\mathbf{0}$ is a $k$ dimesnional zero. Then it follows that $M_{f} s_{f}=\left(E^{\dagger}\right)^{-1} \hat{M} \hat{s}=0$. So for every bosonic solution $s^{i}$ we have the corresponding fermionic solution $s_{f}^{i}=E \hat{s}^{i}$. Of course, it goes other way also: for every fermionic solution $s_{f}^{i}, P E^{-1} s_{f}^{i}$, where $P$ is the projector that projects to the first $(k+2)$ components, will be a bosonic solution. By 
supersymmetric boundary condition, it is meant that for every acceptable bosonic solution the corresponding fermionic solution is also acceptable (and of course this implies the other way also). Now, let us consider $G_{b}^{>}\left(r, r^{\prime}\right)$. Near $r=\infty$ this will be linear combinations of bosonic solutions that are acceptable at $r=\infty$. Then, $G_{f}^{>}\left(r, r^{\prime}\right)=E(r) G_{b}^{>}\left(r, r^{\prime}\right) E^{\dagger}\left(r^{\prime}\right)$. As a function of $r$ and $r^{\prime}$ this will be linear combinations of fermionic solutions of $M_{f}$ and its conjugate, respectively. If boundary condition are supersymmetric then it is clear $G_{f}^{>}\left(r, r^{\prime}\right)$ will be the correct fermionic Green's function. If the boundary conditions are not supersymmetric then it must be that there is some bosonic solution, say $s_{b}^{1}$, which is not acceptable at $r=\infty$ but the corresponding fermionic solution $s_{f}^{1}$ is acceptable. So in $G_{f}^{>}\left(r, r^{\prime}\right)=E(r) G_{b}^{>}\left(r, r^{\prime}\right) E^{\dagger}\left(r^{\prime}\right)$ one will have to start with a "bosonic Greens function" which as a function of $r$ involves $s_{b}^{1}$ in order to get acceptable fermionic Green's function. However, the acceptable bosonic Green's function will be different as it should not involve $s_{b}^{1}$ as a function of $r$.

Secondly, note that $E(r)$ and $E^{\dagger}\left(r^{\prime}\right)$ are differential operators. So, in the definition of $G_{f}$ above the $E(r)$ appearing on the left is a differential operator that acts on the argument $r$ of $\widetilde{G}_{1,2}\left(r, r^{\prime}\right)$ and $\widetilde{G}_{1,2}^{\prime}$, while $E^{\dagger}\left(r^{\prime}\right)$ appearing on the right is a differential operator in variable $r^{\prime}$ and acts on the argument $r^{\prime}$ of $\widetilde{G}_{1,2}\left(r, r^{\prime}\right)$ and $\widetilde{G}_{1,2}^{\prime}\left(r, r^{\prime}\right)$ (with $\frac{d}{d r^{\prime}} \rightarrow$ $\left.-\frac{d}{d r^{\prime}}\right)$. One can see this as follows:

We start with the inhomogeneous equation

$$
M_{f}\left(\begin{array}{c}
Q X_{0} \\
c \\
X_{1}^{\prime}
\end{array}\right)=\left(\begin{array}{c}
h_{1} \\
h_{2} \\
h_{3}
\end{array}\right)
$$

Then we want to show that the solution of the above equation is

$$
\left(\begin{array}{c}
Q X_{0}(r) \\
c(r) \\
X_{1}^{\prime}(r)
\end{array}\right)=\int_{r^{\prime}>r} d r^{\prime} G_{f}^{<}\left(r, r^{\prime}\right)\left(\begin{array}{c}
h_{1}\left(r^{\prime}\right) \\
h_{2}\left(r^{\prime}\right) \\
h_{3}\left(r^{\prime}\right)
\end{array}\right)+\int_{r^{\prime}<r} d r^{\prime} G_{f}^{>}\left(r, r^{\prime}\right)\left(\begin{array}{c}
h_{1}\left(r^{\prime}\right) \\
h_{2}\left(r^{\prime}\right) \\
h_{3}\left(r^{\prime}\right)
\end{array}\right),
$$

with the functions $G_{f}^{<}\left(r, r^{\prime}\right)$ and $G_{f}^{>}\left(r, r^{\prime}\right)$ given in (2.14) and (2.15), respectively. To prove this we first integrate $\frac{d}{d r^{\prime}}$ appearing in $E^{\dagger}$ in $G_{f}^{<}$and $G_{f}^{>}$by parts. We get two contributions:

1) The boundary term

$$
\begin{aligned}
E_{1}(r)\left(\left.\partial_{r} \hat{G}_{f}^{>}\left(r, r^{\prime}\right)\right|_{r^{\prime}=r-}-\left.\partial_{r} \hat{G}_{f}^{<}\left(r, r^{\prime}\right)\right|_{r^{\prime}=r+}\right) E_{1}^{\dagger}(r)\left(\begin{array}{c}
h_{1}(r) \\
h_{2}(r) \\
h_{3}(r)
\end{array}\right) & =\left(\begin{array}{c}
0 \\
0 \\
\frac{1}{\gamma_{1}} D^{-1} C_{1}\left(A_{1}^{b(2)}\right)^{-1} C_{1}^{\dagger} D^{-1} h_{3}(r)
\end{array}\right), \\
& =\left(\begin{array}{c}
0 \\
0 \\
D^{-1} h_{3}(r)
\end{array}\right)
\end{aligned}
$$

where in the first equality we have used the discontinuity relation $(2.12)$ and $A_{1}^{b(2)}(r)$ is the matrix coefficient of the second order differential operator $A_{1}^{b}(r)$. In the second 
equality we use the fact that the first order derivative in $E(r)$ and $E(r)^{\dagger}$ appears only in the off-diagonal blocks involving $C(r)$ and $C(r)^{\dagger}$ where

$$
C(r)=C_{1}(r) \frac{d}{d r}+C_{0}(r), \quad \text { and } \quad C(r)^{\dagger}=-\frac{d}{d r} C_{1}(r)^{\dagger}+C_{0}(r)^{\dagger} .
$$

The second equality in (2.19) can be argued as follows. $C$ and $K$ are $k \times(k+1)$ matrix and $1 \times(k+1)$ matrices. We can define a $k$ dimensional space $V_{1}$ and a one-dimensional space $V_{2}$ which satisfy the conditions:

$$
K V_{1}=0, \quad C_{1} V_{2}=0 .
$$

The fact that $V_{2}$ is one- dimensional follows from the non-degeneracy of coefficient of the second derivative term in $A_{1}$ namely $\gamma_{1} A_{1}^{(2)}=-K^{\dagger} A_{22}^{(2)} K+C_{1}^{\dagger} D^{-1} C_{1}$. Now we can choose a basis for $(k+1)$ dimensional space (represented as $(k+1)$ dimesnional row vector) such that the first $V_{1}$ occupies the first $k$ elements while $V_{2}$ the last element. Then the $C_{1}=\left(c_{1} \mathbf{0}\right)$ where $c_{1}$ is a non-degenerate $(k \times k)$ matrix and $\mathbf{0}$ is the $k$ dimensional null vector. Furthermore $K$ is a $(k+1)$ dimensional row vector with the first $k$ elements being zero. It follows that $\left.\gamma_{1} A_{1}^{(2)}\right|_{V_{2}}=-K^{\dagger} A_{22}^{(2)} K$ and $\left.\gamma_{1} A_{1}^{(2)}\right|_{V_{1}}=c_{1}^{\dagger} D^{-1} c_{1}$. The last equality implies that $\left.\left(\gamma_{1} A_{1}^{(2)}\right)^{-1}\right|_{V_{1}}=c_{1}^{-1} D\left(c_{1}^{\dagger}\right)^{-1}$. Thus in this basis we have:

$$
\begin{aligned}
\frac{1}{\gamma_{1}} D^{-1} C_{1}\left(A_{1}^{(2)}\right)^{-1} C_{1}^{\dagger} D^{-1} & =\frac{1}{\gamma_{1}} D^{-1} c_{1}\left(\left.A_{1}^{(2)}\right|_{V_{1}}\right)^{-1} c_{1}^{\dagger} D^{-1}, \\
& =D^{-1},
\end{aligned}
$$

which proves (2.19). Applying $M_{f}$ on (2.19) gives:

$$
\left(\begin{array}{c}
C^{\dagger} D^{-1} h 3 \\
0 \\
h_{3}(r)
\end{array}\right)
$$

2) The bulk term

This is the same as (2.18) except that $E^{\dagger}$ appeaing $G_{f}$ and $G_{f}^{\prime}$ act now to the right i.e. on the source. The bulk term can be rewritten as

$$
E(r)\left(\int_{r^{\prime}>r} d r^{\prime} \hat{G}^{<}\left(r, r^{\prime}\right)+\int_{r^{\prime}<r} d r^{\prime} \hat{G}^{>}\left(r, r^{\prime}\right)\right) E^{\dagger}\left(r^{\prime}\right)\left(\begin{array}{l}
h_{1}\left(r^{\prime}\right) \\
h_{2}\left(r^{\prime}\right) \\
h_{3}\left(r^{\prime}\right)
\end{array}\right) .
$$

This is so because the boundary term that appears in pulling the differential operator $E$ outside the integral vanishes due the discontinuity relation (2.12).

Now, let us apply $M_{f}(r)=\left(E^{\dagger}(r)\right)^{-1} \hat{M}(r) E(r)^{-1}$ on the bulk term (2.24). First of all $E(r)^{-1}$ removes $E(r)$ in $(2.24)$. The action of the operator $\hat{M}$ on $\hat{G}^{<}\left(r, r^{\prime}\right)$ and $\hat{G}^{>}\left(r, r^{\prime}\right)$ vanishes since $r \neq r^{\prime}$. So, the only possible contribution can come when 
one of the derivatives $\frac{d}{d r}$ in $\hat{M}$ acts on the limits of the integrations. Using the discontinuity relations (2.12) one can show that this results in

$$
\left(E^{\dagger}\right)^{-1}\left(\begin{array}{lll}
1 & 0 & 0 \\
0 & 1 & 0 \\
0 & 0 & 0
\end{array}\right) E^{\dagger}(r)\left(\begin{array}{l}
h_{1}(r) \\
h_{2}(r) \\
h_{3}(r)
\end{array}\right)=\left(\begin{array}{c}
h_{1}(r)-C^{\dagger} D^{-1} h 3(r) \\
h_{2}(r) \\
0
\end{array}\right)
$$

where we have used the explicit form of $\left(E^{\dagger}\right)^{-1}$

$$
\left(E^{\dagger}\right)^{-1}=\left(\begin{array}{ccc}
1-f^{-1, \dagger} K^{\dagger} & C^{\dagger} D^{-1} \\
0 & f^{-1, \dagger} & 0 \\
0 & 0 & 1
\end{array}\right)
$$

Adding the two contributions (2.23) and (2.25), one finds that $M_{f}$ acting on the proposed solution (2.18) indeed reproduces the source.

\subsection{Variation of one loop determinant}

Now one can compute the variation of the one loop determinant (2.1) with respect to $\alpha$

$$
\delta_{\alpha} \ln Z_{1 \text {-loop }}(\alpha)=\frac{1}{2} \int_{0}^{\infty} d r \lim _{r^{\prime} \rightarrow r+} \operatorname{tr}\left(\delta_{\alpha} M_{f}(r) G_{f}^{<}\left(r, r^{\prime}\right)-\delta_{\alpha} M_{b}(r) G_{b}^{<}\left(r, r^{\prime}\right)\right) .
$$

Here "tr" is just the matrix trace and $\delta_{\alpha} \equiv \frac{\delta}{\delta \alpha}$. In the above, we have taken the limit $r^{\prime} \rightarrow r_{+}$. Had we taken the limit $r^{\prime} \rightarrow r_{-}$, the fermionic and bosonic Greens functions will be replaced by $G_{f}^{>}$and $G_{b}^{>}$, respectively but we will see later that the final result does not change. The fermionic part of the variation after using the form of $M_{f}$ and $G_{f}$ and some algebra, is

$$
\begin{aligned}
\operatorname{tr}\left(\delta_{\alpha} M_{f}(r) G_{f}^{<}\left(r, r^{\prime}\right)\right)= & \operatorname{tr} \frac{1}{\gamma_{1}}\left(\delta_{\alpha} A_{11}(r) \widetilde{G}_{1}\left(r, r^{\prime}\right)-\delta_{\alpha}\left(K^{\dagger} A_{22}(r) K\right) \widetilde{G}_{1}\left(r, r^{\prime}\right)\right. \\
& -\delta_{\alpha} B(r) D^{-1}(r) C(r) \widetilde{G}_{1}\left(r, r^{\prime}\right)-\delta_{\alpha} C(r) \widetilde{G}_{1}\left(r, r^{\prime}\right) C^{\dagger}\left(r^{\prime}\right) D^{-1}\left(r^{\prime}\right) \\
& \left.+\delta_{\alpha} D(r) D^{-1}(r) C(r) \widetilde{G}_{1}\left(r, r^{\prime}\right) C^{\dagger}\left(r^{\prime}\right) D^{-1}\left(r^{\prime}\right)\right) \\
& +\operatorname{tr} \frac{1}{\gamma_{2}} \delta_{\alpha}\left(A_{22}(r)\right) f(r) \widetilde{G}_{2}\left(r, r^{\prime}\right) f^{\dagger}\left(r^{\prime}\right), \\
= & \operatorname{tr}\left(\delta_{\alpha} A_{1}^{b}(r) \widetilde{G}_{1}\left(r, r^{\prime}\right)+f^{-1, \dagger}(r) \delta_{\alpha} A_{2}^{b}(r) \widetilde{G}_{2}\left(r, r^{\prime}\right) f^{\dagger}\left(r^{\prime}\right)\right) \\
& +\operatorname{tr} \frac{1}{\gamma_{1}}\left(B(r) \delta_{\alpha}\left(D^{-1}(r) C(r)\right) \widetilde{G}_{1}\left(r, r^{\prime}\right)-\delta_{\alpha} C(r) \widetilde{G}_{1}\left(r, r^{\prime}\right) C^{\dagger}\left(r^{\prime}\right) D^{-1}\left(r^{\prime}\right)\right. \\
& \left.+\delta_{\alpha} D(r) D^{-1}(r) C(r) \widetilde{G}_{1}\left(r, r^{\prime}\right) C^{\dagger}\left(r^{\prime}\right) D^{-1}\left(r^{\prime}\right)\right) .
\end{aligned}
$$

In the above we have used the relations (2.7) and also the fact that $G_{1}\left(r, r^{\prime}\right)$ and $G_{2}\left(r, r^{\prime}\right)$ are Green's function for the kinetic operators $A_{1}^{b}(r)$ and $A_{2}^{b}(r)$, respectively. Thus, the 
fermionic contributions to the variation $(2.27)$ is

$$
\begin{aligned}
\lim _{r^{\prime} \rightarrow r+} \operatorname{tr}\left(\delta_{\alpha} M_{f}(r) G_{f}\left(r, r^{\prime}\right)\right)= & \operatorname{tr}\left(\delta_{\alpha} A_{2}^{b}(r) \widetilde{G}_{2}(r, r)+\delta_{\alpha} A_{1}^{b}(r) \widetilde{G}_{1}(r, r)\right) \\
& +\operatorname{tr} \frac{1}{\gamma_{1}}\left(C^{\dagger}(r) \delta_{\alpha}\left(D^{-1}(r) C(r)\right) \widetilde{G}_{1}(r, r)\right. \\
& \left.-\delta_{\alpha}\left(D^{-1}(r) C(r)\right) \widetilde{G}_{1}(r, r) C^{\dagger}(r)\right) .
\end{aligned}
$$

Now, we see that the first two terms in the above equation cancel the bosonic variation if and only if the boundary conditions are consistent with supersymmetry i.e. when the fermionic Green's function is contructed from the bosonic Green's function (2.9) and (2.10). In this case, we are finally left with

$$
\delta_{\alpha} \ln Z_{1 \text {-loop }}(\alpha)=\frac{1}{2} \int_{0}^{\infty} d r \operatorname{tr} \frac{1}{\gamma_{1}}\left(C^{\dagger} \delta_{\alpha}\left(D^{-1} C\right) G_{1}(r, r)-\delta_{\alpha}\left(D^{-1} C\right) G_{1}(r, r) C^{\dagger}\right),
$$

where the differential operators $C$ and $C^{\dagger}$ appearing on the left and right of $G_{1}$ act on respectively the first and second arguments of $G_{1}$. We can now move the operator $C^{\dagger}$ appearing on the right of $G_{1}$ in the second term of (2.30) to the left of $G_{1}$ by using cyclicity of matrix trace as well as an integration by part. This results in a bulk term which cancels with the first term and a boundary term. Thus, the variation of the one loop determinant becomes

$$
\delta_{\alpha} \ln Z_{1 \text {-loop }}(\alpha)=-\frac{1}{2} \operatorname{tr} \frac{1}{\gamma_{1}}\left(\left.C_{1}^{\dagger}(r) \delta_{\alpha}\left(D^{-1}(r) C(r)\right) G_{1}(r, r)\right|_{r \rightarrow 0} ^{r \rightarrow \infty} .\right.
$$

Note that the operator $C(r)$ acts only on the first argument of the Green's function. Thus, we find that if the fermionic Green's function are related to the bosonic Green's function as in (2.14) and (2.15), the variation of the one loop determinant receives contribution only from the boundary. Moreover, to evaluate the boundary term, we just need to know the bosonic Green's function $G_{1}\left(r, r^{\prime}\right)$. This is one of the most important results of our paper.

Now, if it turns out that the $C$ is independent of $\alpha$ (as we will see in the examples of $\mathrm{AdS}_{2} \times \mathrm{S}^{1}$ ) or its $\alpha$ dependence is subleading near each boundary (we have also observed this in other examples [20]), then from (2.7) we see that $D^{-1}=\gamma_{1} D_{0}^{-1}$, where $D_{0}$ is independent of $\alpha$ (at least near each boundary). Using the relation $\frac{1}{\gamma_{1}}=Q^{2}$ we then conclude that

$$
\delta_{\alpha} \ln Z_{1 \text {-loop }}(\alpha)=\left.\frac{1}{2}\left(\delta_{\alpha} \ln Q^{2}\right) \operatorname{Tr}\left(C_{1}^{\dagger} D_{0}^{-1} C\right) G_{1}(r, r)\right|_{r \rightarrow 0} ^{r \rightarrow \infty}
$$

The above result (2.32) we arrived at by taking the limit $r^{\prime} \rightarrow r^{+}$. If we had taken the other limit $r^{\prime} \rightarrow r^{-}$, we would end up with the same expression as above but with $G_{1}$ replaced by $G_{1}^{\prime}$. The difference between the variations will be

$$
\left.\frac{1}{2}\left(\delta_{\alpha} \ln Q^{2}\right) \operatorname{Tr}\left(C_{1}^{\dagger} D_{0}^{-1} C\right)\left(G_{1}(r, r)-G_{1}^{\prime}(r, r)\right)\right|_{r \rightarrow 0} ^{r \rightarrow \infty} .
$$


Using the discontinuity relation of the Green's function, we find that the above difference becomes

$$
\left.\frac{1}{2}\left(\delta_{\alpha} \ln Q^{2}\right) \operatorname{Tr}\left(C_{1}^{\dagger} D_{0}^{-1} C_{1}\right) \frac{1}{A_{1}^{(2)}(r)}\right|_{r \rightarrow 0} ^{r \rightarrow \infty}=\left.\frac{1}{2}\left(\delta_{\alpha} \ln Q^{2}\right) \operatorname{Tr}_{V_{1}} I_{k}\right|_{r \rightarrow 0} ^{r \rightarrow \infty}=0 .
$$

In the above $I_{k}$ is a $k \times k$ identity matrix. Thus, it is reassuring that the result does not depend on the way one takes the limit $r^{\prime} \rightarrow r$.

It will be interesting to investigate the cases where the $\alpha$ dependence in $C$ is not subleading and its implications on the Green's function method presented above.

\subsection{Calculation of the boundary terms}

Now, we will evaluate the boundary terms (2.31). The boundary term is given in terms of the Green's function of the differential operator $A_{1}^{b}$ which is a $(k+1) \times(k+1)$ matrix second order differential operator. We have stated earlier, as a part of our assumptions (2.7), that the $A_{1}^{b}$ can be expressed in terms of the fermionic operator as $A_{11}-K^{\dagger} A_{22} K-B D^{-1} C$. This is one of the consequences of supersymmetry. Furthermore, the second order derivative term in $A_{1}^{b}$ comes from $K^{\dagger} A_{22} K$ and $B D^{-1} C$. While the former has rank 1 the latter has rank $k$. In order to simplify the computations, we can decompose the $(k+1)$ dimensional space in terms of a $k$ dimensional space $V_{1}$ and a one-dimensional space $V_{2}$ as in (2.21). This means that second derivative part of $K^{\dagger} A_{22} K$ in $A_{1}^{b}$ acts only on $V_{2}$ and that of $B D^{-1} C$, namely $B_{1} D^{-1} C_{1}$ acts only on $V_{1}$. Of course the first order derivative and nonderivative pieces contained in $A_{11}$ and $B D^{-1} C$ will in general act on both $V_{1}$ and $V_{2}$ and therefore, the operator $A_{1}^{b}$ will mix these two spaces through lower order derivative terms. To evaluate the boundary term (2.31) we will make the following assumptions.

1. The leading behaviour of the solutions of $A_{1}^{b}$ near the boundaries, i.e. near $r=0$ and $r=\infty$, is determined by $K^{\dagger} A_{22} K$ on $V_{2}$ and by $B D^{-1} C$ restricted to $V_{1}$. This means that the first order derivative and non-derivative pieces contained in $A_{11}$, $A_{22}$ and $B D^{-1} C$ that mix $V_{1}$ and $V_{2}$ only contribute to subleading orders. We have checked in all the examples we have studied, assumption holds. In fact, our preliminary calculations also indicate that the assumption follows from the general positive definite localising action of the form $S \sim \Psi(Q \Psi)^{\dagger}$. Therefore, to compute the boundary term (2.31) or (2.32), we only need to study the action of $B D^{-1} C$ and the Green's function, $G_{1}\left(r, r^{\prime}\right)$, restricted to the vector space $V_{1}$. That is, the leading contribution to the boundary term comes from the space of solutions of $B D^{-1} C$ (now viewed as $(k \times k)$ matrix operator $)$ on $V_{1}$.

2. The Greens fn for $A_{1}^{b}$ exists. This, taking into account assumption (1), implies that of the $2 k$ solutions of $B D^{-1} C$ on $V_{1}$ near the boundary at least $k$ solutions satisfy the boundary conditions. Similarly, it implies that of the 2 solutions of $K^{\dagger} A_{22} K$ on $V_{2}$ at least one solution satisfies the boundary condition. 
3. $A_{1}^{b}$ has no zero modes. ${ }^{5}$ This means that there are precisely $k$ solutions to $B D^{-1} C$ on $V_{1}$ and 1 solution of $K^{\dagger} A_{22} K$ on $V_{2}$ that are allowed near each of the boundaries and that none of the allowed $k$ solutions near one boundary, when analytically continued to the other boundary satisfies the corresponding boundary condition.

In the following, by a slight abuse of notation, we will denote by $C$ and $B\left(=C^{\dagger}\right)$ their restrictions to $V_{1}$ i.e. they will be represented (by a suitable change of basis) as $(k \times k)$ matrix operators, unless stated explicitly otherwise. Similarly, we will denote the Green's function of $A_{1}^{b}$ restricted to $V_{1}$ by $G_{1}\left(r, r^{\prime}\right)$ for $r<r^{\prime}$ and $G_{1}^{\prime}\left(r, r^{\prime}\right)$ for $r>r^{\prime}$ and both will be a $k \times k$ matrix.

Now, the assumption (1) could have been relaxed. Of course even if this assumption is not valid in some cases, one can carry out the boundary analysis of the Green's functions and compute the boundary term in the $\alpha$ variation above in each case separately, but this assumption will allow us to obtain a general formula for the boundary term and relate it to the index of the differential operator $C$.

We begin with $2 k$ linearly independent solutions of $A_{1}^{b}$ (now viewed as $(k \times k)$ matrix operator) on $V_{1}$. Let us denote by $S$ a $(k \times 2 k)$ matrix where the $2 k$ columns label the $2 k$ different solutions and let $\eta$ be a diagonal $(2 k \times 2 k)$ matrix with entries -1 for the allowed solutions and +1 for the ones that are not allowed. From the assumptions (2) and (3), there are $k$ solutions each with +1 and -1 eigenvalues of $\eta$. Thus, $\frac{1}{2}(1-\eta)$ and $\frac{1}{2}(1+\eta)$ are projections operator which will project the solutions matrix $S$ into the acceptable and non-acceptable solutions near each boundary. Furthermore, the leading behaviour of the solution $S$ agrees with the leading behaviour of the solution of $B D^{-1} C$ restricted on $V_{1}$. We define the Green's function to be

$$
\begin{aligned}
G_{1}\left(r, r^{\prime}\right) & =\frac{1}{2} S(r)(1-\eta) X\left(r^{\prime}\right), & & \text { for } \quad r<r^{\prime}, \\
G_{1}^{\prime}\left(r, r^{\prime}\right) & =\left(\frac{1}{2} S(r)(1+\eta)+\cdots\right) X\left(r^{\prime}\right), & & \text { for } \quad r>r^{\prime} .
\end{aligned}
$$

Here $X\left(r^{\prime}\right)$ is an unknown $(2 k \times k)$ matrix such that $(1-\eta) X\left(r^{\prime}\right)$ is admissible at the other boundary i.e. at $r \rightarrow \infty$ and $(1+\eta) X\left(r^{\prime}\right)$ satisfy the allowed boundary condition at the first boundary i.e. at $r=0$. The dots in the second equation above denote combinations of allowed solutions i.e. of the form $Y_{1} \frac{1}{2} S(r)(1-\eta) Y_{2}(1+\eta)$ where $Y_{1}$ and $Y_{2}$ are some constant (i.e. independent of $r$ ) matrices. $Y_{1}$ and $Y_{2}$ are determined by requiring that the combination $\left(\frac{1}{2} S(r)+Y_{1} \frac{1}{2} S(r)(1-\eta) Y_{2}\right)(1+\eta)$ are the analytic continuation of allowed solutions near the other boundary. However these dotted terms will be subleading and therefore not be relevant for us and we will drop them in the following. What is important, however, is that the $k$ linearly independent solutions that are admissible at the other boundary, let say at $r=0$, when analytically continued to $r=\infty$, span the $k$ dimensional space $\frac{1}{2} S(r)(1+\eta)$ (of inadmissible solutions) near the first boundary, as is implied by the assumption (3) of the non-existence of zero modes for $A_{1}^{b}$.

\footnotetext{
${ }^{5} A_{2}^{b}$ can have zero mode corresponding to the variation of the saddle point, which happens only for modes that are constant along space orthogonal to $A d S_{2}$ and for which we already have collective coordinate integration. This case will be discussed separately.
} 
Next, we determine $X(r)$. When $r \neq r^{\prime}$ both $G$ and $\hat{G}$ are annihilated by $A_{1}^{b}$. The continuity/discontinuity relations for the Greens function near $r=r^{\prime}$ are:

$$
\begin{aligned}
G_{1}^{\prime}(r, r)-G_{1}(r, r) & =0, \\
\lim _{\epsilon \rightarrow 0} B_{1} D_{0}^{-1} C_{1} \partial_{r}\left(\left.G_{1}^{\prime}\left(r, r^{\prime}\right)\right|_{r^{\prime}=r-\epsilon}-\left.G_{1}\left(r, r^{\prime}\right)\right|_{r^{\prime}=r+\epsilon}\right) & =\mathbf{1} .
\end{aligned}
$$

Here 1 is a $k \times k$ identity matrix. Note that in the second line we have used the fact that second order differential operator $A_{22}$ in $A_{1}^{b}$ does not play a role on the solution in vector space $V_{1}$. Using the continuity equation, the discontinuity equation can also be written as

$$
\lim _{\epsilon \rightarrow 0} B_{1} D_{0}^{-1} C\left(\left.G_{1}^{\prime}\left(r, r^{\prime}\right)\right|_{r^{\prime}=r-\epsilon}-\left.G_{1}\left(r, r^{\prime}\right)\right|_{r^{\prime}=r+\epsilon}\right)=\mathbf{1} .
$$

Using the expressions for the Green's function given in (2.35), we write the two equations in $(2.36)$ as a matrix equation for $X(r)$

$$
W(r) \eta X(r)=\left(\begin{array}{l}
0 \\
\mathbf{1}
\end{array}\right)
$$

where

$$
W=\left(\begin{array}{c}
S(r) \\
B_{1} D_{0}^{-1} C S(r)
\end{array}\right)=\left(\begin{array}{cc}
s(r) & \tilde{s}(r) \\
B_{1} D_{0}^{-1} C s(r) & B_{1} D_{0}^{-1} C \tilde{s}(r)
\end{array}\right) .
$$

In the above we have split $k \times 2 k$ matrix $S$ as $S=(s(r) \widetilde{s}(r))$, where $s(r)=\left\{s^{i}(r)\right\}$ and $\widetilde{s}(r)=\left\{\widetilde{s}^{i}(r)\right\}$, for $i=1, \ldots, k$ are solutions of $A_{1}^{b}$. Thus we obtain

$$
X(r)=\eta W^{-1}\left(\begin{array}{l}
0 \\
\mathbf{1}
\end{array}\right) .
$$

Note that the inverse of $W$ exist because the determinant of $W$ is determinant of $B_{1} D_{0}^{-1} C_{1}$ times the Wronskian and hence non-zero because of our assumptions. Since to evaluate the boundary term (2.32), we just need to know the asymptotic form of the Green's function, we therefore, only require the asymptotic form of $X(r)$ at each boundary. To begin with we first consider the analysis near the boundary i.e. $r=0$. Without loss of generality, we can assume that the set of solutions $\left\{s^{i}(r)\right\}$, for $i=1, \ldots, k$ belong to the kernel of $C$ near $r=0$. In this case near $r=0$, we have

$$
\lim _{r \rightarrow 0} W^{-1}=\left(\begin{array}{cc}
s^{-1}-s^{-1} \tilde{s}\left(B_{1} D_{0}^{-1} C \tilde{s}(r)\right)^{-1} \\
0 & \left(B_{1} D_{0}^{-1} C \tilde{s}(r)\right)^{-1}
\end{array}\right),
$$

where we have used the fact that $B_{1} D_{0}^{-1} C s(r)=0$. In this case near the boundary $r=0$, the solution (2.40) becomes

$$
\begin{aligned}
X(r) & =\eta_{0}\left(\begin{array}{cc}
s^{-1}(r)-s^{-1}(r) \tilde{s}(r)\left(B_{1} D_{0}^{-1} C \tilde{s}(r)\right)^{-1} \\
0 & \left(B_{1} D_{0}^{-1} C \tilde{s}(r)\right)^{-1}
\end{array}\right)\left(\begin{array}{l}
0 \\
\mathbf{1}
\end{array}\right), \\
& =\eta_{0}\left(\begin{array}{c}
-s^{-1}(r) \tilde{s}(r)\left(B_{1} D_{0}^{-1} C \tilde{s}(r)\right)^{-1} \\
\left(B_{1} D_{0}^{-1} C \tilde{s}(r)\right)^{-1}
\end{array}\right),
\end{aligned}
$$


where with this ordering of the solutions in $S(r)$, so that first $k$ column belongs to the Kernel of $C$ near $r=0$, the corresponding projector is $\eta_{0}$. Using the above equation we can obtain the Green's function for $r<r^{\prime}$ near the boundary $r=r^{\prime}=0$ as

$$
G_{1}\left(r, r^{\prime}\right)=-\frac{1}{2}(s(r) \tilde{s}(r))\left(1-\eta_{0}\right)\left(\begin{array}{c}
-s^{-1}\left(r^{\prime}\right) \tilde{s}\left(r^{\prime}\right)\left(B_{1} D_{0}^{-1} C \tilde{s}\left(r^{\prime}\right)\right)^{-1} \\
\left(B_{1} D_{0}^{-1} C \tilde{s}\left(r^{\prime}\right)\right)^{-1}
\end{array}\right) .
$$

Now, we can compute the boundary term at $r=0$ by using (2.32) and the expression for $G\left(r, r^{\prime}\right)$ from (2.35) and (2.43) and the result is

$$
\begin{aligned}
\lim _{r \rightarrow 0} \operatorname{Tr}\left(\left.B_{1} D_{0}^{-1} C G_{1}\left(r, r^{\prime}\right)\right|_{r^{\prime} \rightarrow r}\right) & =-\frac{1}{2} \operatorname{Tr}\left[\left(0 B_{1} D_{0}^{-1} C \tilde{s}(r)\right)(1-\eta)\left(\begin{array}{c}
-s^{-1}(r) \tilde{s}(r)\left(B_{1} D_{0}^{-1} C \tilde{s}(r)\right)^{-1} \\
\left(B_{1} D_{0}^{-1} C \tilde{s}(r)\right)^{-1}
\end{array}\right)\right], \\
& =-\frac{1}{2} \operatorname{Tr}\left[(1-\eta)\left(\begin{array}{cc}
0-s^{-1}(r) \tilde{s}(r) \\
0 & 1
\end{array}\right)\right], \\
& =-(k-\ell) .
\end{aligned}
$$

where $\ell$ is the number of admissible solutions at $r=0$ that are in the Kernel of $C$.

We can repeat the same analysis at $r=\infty$. The difference now is that for $r>r^{\prime}$ the Green's function must involve solutions that are admissible near $r=\infty$. Let the corresponding projector be $\frac{1}{2}\left(1-\eta_{\infty}\right)$. Then we have the Green's function as in $(2.35)$ with $\eta \rightarrow-\eta_{\infty}$. We can repeat the above analysis except that we assume that our set of solutions to $S(r)=\left(s^{\prime}(r) \widetilde{s}^{\prime}(r)\right)$ such that the first $k$ column belongs to the Kernel of $C$ near $r=\infty$. Following the same steps as above, we get the contribution to the boundary term near $r=\infty$

$$
\begin{array}{r}
-\frac{1}{2} \operatorname{Tr}\left[\left(1+\eta_{\infty}\right)\left(\begin{array}{cc}
0 & -s^{-1}(r) \tilde{s}(r) \\
0 & \mathbf{1}
\end{array}\right)\right] \\
=-\ell^{\prime},
\end{array}
$$

where $\ell^{\prime}$ is the number of admissible solutions in the set $\left\{s^{\prime}(r)\right\}$ at $r=\infty$ that are in the Kernel of $C$. Note in the above we have used the fact that the first $k \times k$ block of $\frac{1}{2}\left(1+\eta_{\infty}\right)$ has $\ell^{\prime}$ zeros.

Taking the difference between the contribution at $r=\infty$ and at $r=0$ one ends up with the simple result

$$
\left.\mathrm{BT} \equiv \operatorname{Tr}\left(C_{1}^{\dagger} D_{0}^{-1} C\right) G_{1}(r, r)\right|_{r \rightarrow 0} ^{r \rightarrow \infty}=\left(k-\ell-\ell^{\prime}\right) .
$$

Finally combining (2.46) and (2.32) we obtain our main result

$$
\delta_{\alpha} \ln Z_{1 \text {-loop }}(\alpha)=\frac{1}{2}\left(\delta_{\alpha} \ln Q^{2}\right)\left(k-\ell-\ell^{\prime}\right) .
$$

Here we again recall the various integers that occur in this expression.

1. $k+1$ is the integer that defines dimension of the bosonic space $X_{0}$.

2. $\ell, \ell^{\prime}$ are the number of admissible solutions of the first order equations $C s(r)=0$ at the origin and at asymptotic infinity of AdS, respectively. 
Note that the above result (2.47) is obtained for each Kaluza Klein mode. Therefore, to obtain the complete contribution to the variation of the one loop determinant we need to sum over KK modes labelled by $\vec{n}$

$$
\delta_{\alpha} \ln Z_{1-\text { loop }}^{\text {total }}(\alpha)=\frac{1}{2} \sum_{\vec{n}}\left(\delta_{\alpha} \ln Q_{\vec{n}}^{2}\right)\left(k-\ell_{\vec{n}}-\ell_{\vec{n}}^{\prime}\right) .
$$

\subsection{Connection to index of the operator $C$}

In this section we will show that the result of the boundary term (2.46) is an index of the first order differential operator $\mathcal{C}=\left.C\right|_{V_{1}}$. To show this we start with the fact that the operator $\left.A_{1}^{b}(r)\right|_{V_{1}}$, whose one loop determinant we are interested in to compute, asymptotically approaches $\mathcal{C}^{\dagger} D^{-1} \mathcal{C}$. Therefore, a solution of the operator $\left.A_{1}^{b}(r)\right|_{V_{1}} s=0$ near each boundary either belong to the solution space $S(\mathcal{C})$ of the operator $\mathcal{C}$ or the solutions space $S\left(\mathcal{C}^{\dagger}\right)$ of $\mathcal{C}^{\dagger}$ which is a subset of the image of the operator $\mathcal{C}$. We start with the solution space $S(\mathcal{C})$. A solution in $S(\mathcal{C})$ has asymptotic behaviour $r^{\gamma}$ and $e^{\hat{\gamma} r}$ near $r \rightarrow 0$ and $r \rightarrow \infty$, respectively. Let this set be $\Gamma(\mathcal{C})=\left\{\gamma_{1}, \ldots, \gamma_{k}\right\}$ and $\hat{\Gamma}(\mathcal{C})=\left\{\hat{\gamma}_{1}, \ldots, \hat{\gamma}_{k}\right\}$. The rest $k$ solutions of $\left.A_{1}^{b}(r)\right|_{V_{1}}$ correspond to the set $S\left(\mathcal{C}^{\dagger}\right)$, the space of solution of $\mathcal{C}^{\dagger}$, and the corresponding set of the asymptotic behaviour be $\Gamma\left(\mathcal{C}^{\dagger}\right)=\left\{\gamma_{1}^{*}, \ldots, \gamma_{k}^{*}\right\}$ and $\hat{\Gamma}\left(\mathcal{C}^{\dagger}\right)=\left\{\hat{\gamma}_{1}^{*}, \ldots, \hat{\gamma}_{k}^{*}\right\}$. Now given these sets near each boundary the differential operators $\mathcal{C}$ and $\mathcal{C}^{\dagger}$ can be diagonalised. Near $r \rightarrow 0$ differential operators $\mathcal{C}$ and $\mathcal{C}^{\dagger}$ can be brought to the form ${ }^{6}$

$$
\mathcal{C}=I_{k} \frac{d}{d r}+\frac{1}{r} \mathcal{C}_{0}^{\text {diag. }}, \quad \mathcal{C}^{\dagger}=-I_{k} \frac{d}{d r}+\frac{1}{r} \mathcal{C}_{0}^{\dagger \text { diag. }},
$$

and near $r \rightarrow \infty$ differential operators $\mathcal{C}$ and $\mathcal{C}^{\dagger}$ can be brought to the form

$$
\mathcal{C}=I_{k} \frac{d}{d r}+\mathcal{C}_{\infty}^{\text {diag. }}, \quad \mathcal{C}^{\dagger}=-I_{k} \frac{d}{d r}+\mathcal{C}_{\infty}^{\dagger \text { diag. }}
$$

Here $\mathcal{C}_{0}^{\text {diag. }}\left(\mathcal{C}_{0}^{\dagger \text { diag. }}\right)$ and $\mathcal{C}_{\infty}^{\text {diag. }}\left(\mathcal{C}_{\infty}^{\dagger \text { diag. }}\right)$ are constant $k \times k$ matrices with diagonal entries given by $\Gamma(\mathcal{C})\left(\Gamma\left(\mathcal{C}^{\dagger}\right)\right)$ and $\hat{\Gamma}(\mathcal{C})\left(\hat{\Gamma}\left(\mathcal{C}^{\dagger}\right)\right)$, respectively.

Next, we consider an operator $\mathcal{C}^{\prime}\left(\mathcal{C}^{\dagger}\right)$ which is continuously connected to $\mathcal{C}\left(\mathcal{C}^{\dagger}\right)$ and is defined globally for every value of $r$. This operator has the form

$$
\mathcal{C}^{\prime}=I_{k} \frac{d}{d r}+\mathcal{C}_{0}^{\prime}(r), \quad \mathcal{C}^{\prime \dagger}=-I_{k} \frac{d}{d r}+\mathcal{C}_{0}^{\prime \dagger}(r)
$$

The non derivative term $\mathcal{C}_{0}^{\prime}\left(\mathcal{C}_{0}^{\prime \dagger}\right)$ is such that the operator $\mathcal{C}^{\prime}\left(\mathcal{C}^{\prime \dagger}\right)$ is a $k \times k$ diagonal first order differential operator for every value of $r$ and near the boundary it approaches the asymptotic form (2.49) and (2.50) of the differential operator $\mathcal{C}\left(\mathcal{C}^{\dagger}\right)$. Thus, $\mathcal{C}^{\prime}\left(\mathcal{C}^{\prime \dagger}\right)$ is an interpolating operator between the asymptotic (2.49) and (2.50). Since the operator $\mathcal{C}^{\prime}$ is continuously connected to the operator $\mathcal{C}$, we expect that the index of $\mathcal{C}^{\prime}$ to be same as that of the operator $\mathcal{C}$.

\footnotetext{
${ }^{6}$ Note that one can always put the operator $\mathcal{C}$ and $\mathcal{C}^{\dagger}$ of the form (2.49) and (2.50) without the non derivative term being diagonal.
} 
Now we will compute the index of the operator $\mathcal{C}^{\prime}$. Let $S\left(\mathcal{C}^{\prime}\right)$ be the space of solutions of matrix differential operator $\mathcal{C}^{\prime}$. Since $\mathcal{C}^{\prime}$ is a $k \times k$ first order matrix differential operator, we expect the dimension for the space of solutions to be $\operatorname{dim} S\left(\mathcal{C}^{\prime}\right)=k$. We consider two spaces, $S_{1}\left(\mathcal{C}^{\prime}\right) \subset S\left(\mathcal{C}^{\prime}\right)$ and $S_{2}\left(\mathcal{C}^{\prime}\right) \subset S\left(\mathcal{C}^{\prime}\right)$, where $S_{1}\left(\mathcal{C}^{\prime}\right)$ are the set of solutions which are smooth near $r=0$ and $S_{2}\left(\mathcal{C}^{\prime}\right)$ are the set of solutions which are admissible near $r=\infty$. Since, operators $\mathcal{C}$ and $\mathcal{C}^{\prime}$ have the same asymptotic, therefore, they have the same dimension of the space of admissible solution. Thus, $\operatorname{dim} S_{1}\left(\mathcal{C}^{\prime}\right)=\ell$ and $\operatorname{dim} S_{2}\left(\mathcal{C}^{\prime}\right)=\ell^{\prime}$. Let the space of Kernel of $\mathcal{C}^{\prime}$ is $\operatorname{Ker}\left(\mathcal{C}^{\prime}\right)$ and its dimension is $s$. The space $\operatorname{Ker}\left(\mathcal{C}^{\prime}\right) \subset S\left(\mathcal{C}^{\prime}\right)$ is the space of solutions which are smooth near $r=0$ as well as admissible near $r=\infty$. Clearly, $\operatorname{Ker}\left(\mathcal{C}^{\prime}\right)=S_{1}\left(\mathcal{C}^{\prime}\right) \cap S_{2}\left(\mathcal{C}^{\prime}\right)$. Furthermore, we expect that there are solutions $\in S\left(\mathcal{C}^{\prime}\right)$ which are neither smooth near $r=0$ nor admissible near $r=\infty$. These solutions belong to the space $\hat{S}\left(\mathcal{C}^{\prime}\right)=S\left(\mathcal{C}^{\prime}\right) / S_{1}\left(\mathcal{C}^{\prime}\right) \cup S_{2}\left(\mathcal{C}^{\prime}\right)$ and the dimension of this space is

$$
\operatorname{dim} \hat{S}\left(\mathcal{C}^{\prime}\right)=k-\ell-\ell^{\prime}+s=k-\ell-\ell^{\prime}+\operatorname{dim} \operatorname{Ker}\left(\mathcal{C}^{\prime}\right) .
$$

Next, we argue that for every solution belonging to $\hat{S}\left(\mathcal{C}^{\prime}\right), \exists$ a solution belonging to $\operatorname{Ker}\left(\mathcal{C}^{\prime} \dagger\right)$. In particular, given a solution in $S\left(\mathcal{C}^{\prime}\right)$ which is neither smooth near $r=0$ nor admissible near $r=\infty$, the existence of Green's function of $A_{1}^{b}(r)$ requires that there exist a solution belonging to the Kernel of $\mathcal{C}^{\dagger}$ which is smooth near $r=0$ and admissible near $r=\infty$. Thus

$$
\operatorname{dim} \operatorname{Ker}\left(\mathcal{C}^{\prime \dagger}\right)-\operatorname{dim} \operatorname{Ker}\left(\mathcal{C}^{\prime}\right)=k-\ell-\ell^{\prime}
$$

The argument goes as follows: let us consider a solution $s_{i} \in \hat{S}\left(\mathcal{C}^{\prime}\right)$ which has asymptotic determined by $\gamma_{i} \in \Gamma(\mathcal{C})$ and $\hat{\gamma}_{i} \in \hat{\Gamma}(\mathcal{C})$ near $r=0$ and $r=\infty$, respectively. Both $\gamma_{i}$ and $\hat{\gamma}_{i}$ correspond to non admissible behaviour. Now we require that near each boundary the Green's function of $\left.A_{1}^{b}(r)\right|_{V_{1}}$ exists. Since, $\left.A_{1}^{b}(r)\right|_{V_{1}}$ asymptote to $\mathcal{C}^{\dagger} D^{-1} \mathcal{C}$, it implies that for every such $\gamma_{i} \in \Gamma(\mathcal{C})$ at $r=0$ there is $\gamma_{i}^{*} \in \Gamma\left(\mathcal{C}^{\dagger}\right)$ and for every such $\hat{\gamma}_{i} \in \hat{\Gamma}(\mathcal{C})$ at $r=\infty$ these is $\gamma_{i}^{*} \in \hat{\Gamma}\left(\mathcal{C}^{\dagger}\right)$, where $\gamma_{i}^{*}$ and $\hat{\gamma}_{i}^{*}$ give rise admissible asymptotic behaviour. Since $\mathcal{C}^{\prime} \dagger$ asymptote to $\mathcal{C}^{\dagger}$ near each boundary, this implies that there exist a solution $\bar{s}_{i}$ of $\mathcal{C}^{\dagger} \dagger$ which has the asymptotic behaviour determined by $\gamma_{i}^{*}$ and $\hat{\gamma}_{i}^{*}$ and is acceptable at both ends. Thus it belongs to the kernel of $\mathcal{C}^{\prime} \dagger$. Furthermore, using the inner product $\left\langle v_{1}, v_{2}\right\rangle=\int d r v_{1}^{\dagger} v_{2}$, one sees that the space $\operatorname{Ker} \mathcal{C}^{\prime} \dagger$ is isomorphic to the space Coker $\mathcal{C}^{\prime}$. Thus

$$
\operatorname{ind}\left(\mathcal{C}^{\prime}\right) \equiv \operatorname{dim} \operatorname{Coker}\left(\mathcal{C}^{\prime}\right)-\operatorname{dim} \operatorname{Ker}\left(\mathcal{C}^{\prime}\right)=k-\ell-\ell^{\prime} .
$$

Since $\mathcal{C}^{\prime}$ is continuously related to $\mathcal{C}$,

$$
\operatorname{ind}(\mathcal{C})=k-\ell-\ell^{\prime}
$$

Thus, the boundary term (2.46) is the index of the operator $\mathcal{C}=\left.C\right|_{V_{1}}$.

\section{Chern-Simons theory on $\operatorname{AdS}_{2} \times S^{1}$ : Greens function approach}

In this section, we revisit the analysis presented in [17]. In [17], we computed the partition function of a non abelian bosonic Chern Simons theory on the metric background

$$
d s^{2}=d \tau^{2}+L^{2}\left(d r^{2}+\sinh ^{2} r d \theta^{2}\right)
$$


where $L$ is some constant, using the supersymmetric localization. This is possible because of the following reason: the supersymmetric completion of a bosonic Chern-Simons action is

$$
S_{\mathrm{C} . \mathrm{S} .}=\int d^{3} x \sqrt{g} \operatorname{Tr}\left[i \varepsilon^{\mu \nu \rho}\left(a_{\mu} \partial_{\nu} a_{\rho}-\frac{2 i}{3} a_{\mu} a_{\nu} a_{\rho}\right)-\tilde{\lambda} \lambda+\frac{i}{2} H \sigma\right] .
$$

Here $\varepsilon^{\mu \nu \rho}=\frac{1}{\sqrt{g}} \epsilon^{\mu \nu \rho}, \quad \epsilon^{\tau \eta \theta}=1$. Also, in order to construct supersymmetric action, we have used the vector multiplet in $\mathcal{N}=2$ theory in Euclidean signature which contains an imaginary scalar $\sigma$, gauge field $a_{\mu}$, an auxiliary scalar field $H$ which is also imaginary and 2 component complex fermions $\lambda$ and $\tilde{\lambda}$. Now, we note that the fermions and scalars in the vector multiplet are purely auxiliary fields as they do not have kinetic terms and therefore, one can integrate them out. Thus the supersymmetric Chern-Simons theory is equivalent to a bosonic Chern-Simons theory.

The analysis in [17] was based on index computation which relies on the boundary conditions being consistent with supersymmetry. These consist of normalizable boundary conditions on the gauge field and non normalizable boundary conditions on fermions following from supersymmetry transformations. We find that the one loop determinant evaluated using the index calculations is given as

$$
Z_{1 \text {-loop }}(\alpha)=\prod_{\rho} \sqrt{\prod_{n \neq 0}(n-i \rho \cdot \alpha) \prod_{p \neq 0}\left(\frac{p}{L}-i \rho \cdot \alpha\right)} .
$$

We will reproduce the above answer in the Green's function approach with normalizable boundary conditions on all fields, including fermions, and find that the above result holds true as long as $L^{2}>\frac{3}{4}$. It would be interesting to understand the significance of the rational number $\frac{3}{4}$.

\subsection{Q-exact deformation and gauge fixing}

Next, we deform the action (3.2) by a $Q$-exact term, $t Q V_{\text {loc }}$. We express the $Q V_{\text {loc }}$ in terms fermion bilinear $\left(\Psi, \Psi_{\mu}\right)$ instead of $(\lambda, \tilde{\lambda})$ which are defined as

$$
\Psi=\frac{i}{2}(\tilde{\epsilon} \lambda+\epsilon \tilde{\lambda}), \quad \Psi_{\mu}=Q_{s} a_{\mu}=\frac{1}{2}\left(\epsilon \gamma_{\mu} \tilde{\lambda}+\tilde{\epsilon} \gamma_{\mu} \lambda\right) .
$$

The fermion bi-linears are convenient for the evaluation of the index. The inverse of the above relations expresses $(\lambda, \tilde{\lambda})$ in terms of $\Psi, \Psi_{\mu}$ as

$$
\lambda=\frac{1}{\tilde{\epsilon} \epsilon}\left[\gamma^{\mu} \epsilon \Psi_{\mu}-i \epsilon \Psi\right], \quad \tilde{\lambda}=\frac{1}{\epsilon \tilde{\epsilon}}\left[\gamma^{\mu} \tilde{\epsilon} \Psi_{\mu}-i \tilde{\epsilon} \Psi\right] .
$$

The supersymmetry transformation of the bi-linears are

$$
\begin{aligned}
Q_{s} \Psi & =\frac{1}{4}(\tilde{\epsilon} \epsilon) H-\frac{i}{2}\left(\tilde{\epsilon} \gamma^{\mu \nu} \epsilon\right) F_{\mu \nu}-\frac{1}{L} \sigma \\
Q_{s} \Psi_{\mu} & =\mathcal{L}_{K} a_{\mu}+D_{\mu} \Lambda
\end{aligned}
$$

where $\Lambda=\widetilde{\epsilon} \epsilon \sigma-K^{\mu} a_{\mu}$. One convenient choice of $V_{\text {loc }}$ is given by

$$
V_{\mathrm{loc}}=\int d^{3} x \sqrt{g} \frac{1}{(\tilde{\epsilon} \epsilon)^{2}} \operatorname{Tr}\left[\Psi^{\mu}\left(Q_{s} \Psi_{\mu}\right)^{\dagger}+\Psi\left(Q_{s} \Psi\right)^{\dagger}\right] .
$$


The bosonic part of the $Q V_{\text {loc }}$ action is given by

$$
\begin{aligned}
Q_{s} V_{\text {loc }\{\text { bosonic }\}=}=\int d^{3} x \sqrt{g} \frac{1}{2(\tilde{\epsilon} \epsilon)^{2}} \operatorname{Tr}\left[\left(Q_{s} \Psi^{\mu}\right)\left(Q_{s} \Psi_{\mu}\right)^{\dagger}+\left(Q_{s} \Psi\right)\left(Q_{s} \Psi\right)^{\dagger}\right] \\
=\int d^{3} x \sqrt{g} \operatorname{Tr}\left[\frac{1}{4} F_{\mu \nu} F^{\mu \nu}-\frac{1}{2 \cosh ^{2} r} D_{\mu}(\cosh r \sigma) D^{\mu}(\cosh r \sigma)\right. \\
\left.-\frac{1}{32}\left(H-\frac{4 \sigma}{L \cosh r}\right)^{2}\right]
\end{aligned}
$$

For a gauge group $G$ with rank $r$, the minimum of the $Q_{s} V_{\text {loc }\{\text { bosonic }\}}$ is parametrized by $r$ real parameters as

$$
a_{\mu}=0, \quad \sigma=\frac{i \alpha}{\cosh r}, \quad H=\frac{4 i \alpha}{L \cosh ^{2} r} .
$$

Here $\alpha$ is a real constant matrix valued in Lie algebra of the gauge group. Furthermore, on this localization background the gauge transformation parameter in supersymmetry algebra reduces to a constant, $\Lambda^{(0)}=i \alpha$.

Next, we need to introduce the gauge fixing Lagrangian. In our case it turns out that the Green's function analysis becomes simpler for the gauge fixing Lagrangian

$$
\mathcal{L}_{g . f .}=\operatorname{Tr} Q\left[i\left(\tilde{c} \cosh ^{2} r+2[\alpha, c]\right) \nabla_{\mu}\left(\frac{1}{\cosh ^{2} r} a^{\mu}\right)+\xi \tilde{c} b\right]
$$

where $Q=Q_{s}+Q_{B}$ and $Q_{B}$ is the BRST transformation. Below we will define the action of the supersymmetry transformations and BRST transformations on all the fields.

Note that the above gauge fixing Lagrangian is different than the one used in [17]. As we will see below, the above choice of the gauge fixing Lagrangian decouples the equations of motion for the fluctuations of the scalar field $\sigma$ with the gauge field fluctuations.

The complete action including the gauge fixing Lagrangian is invariant under BRST transformations on the fields which are given by

$$
\begin{aligned}
& Q_{B} a_{\mu}=D_{\mu} c, \quad Q_{B} \tilde{c}=b, \quad Q_{B} c=\frac{i}{2}\{c, c\}, \quad Q_{B} \tilde{\lambda}=i\{c, \tilde{\lambda}\}, \\
& Q_{B} \lambda=i\{c, \lambda\}, \quad Q_{B} \hat{\sigma}=i[c, \hat{\sigma}], \quad Q_{B} \hat{H}=i[c, \hat{H}], \quad Q_{B} b=0 .
\end{aligned}
$$

Here $a_{\mu}, \hat{\sigma}$ and $\hat{H}$ are fluctuations away from localizing.

We also define the susy transformations for extra fields

$$
Q_{s} c=-\Lambda+\Lambda^{(0)}, \quad Q_{s} b=\mathcal{L}_{K} \tilde{c}+i\left[\Lambda^{(0)}, \tilde{c}\right], \quad Q_{s} \tilde{c}=0
$$

such that the combined transformations generated by $Q=Q_{s}+Q_{B}$ satisfy the algebra

$$
Q^{2}=\mathcal{L}_{K}+\delta_{\Lambda^{(0)}}^{\text {gauge transf. }}
$$


To summarize, the complete transformations of fields under $\hat{Q}$ are given by

$$
\begin{aligned}
Q a_{\mu} & =\Psi_{\mu}+D_{\mu} c, & Q \hat{\sigma} & =Q_{s} \hat{\sigma}+i[c, \hat{\sigma}], \\
Q \Psi_{\mu} & =\mathcal{L}_{K} a_{\mu}+D_{\mu} \Lambda+i\left\{c, \Psi_{\mu}\right\}, & Q \Psi & =\frac{1}{4}(\tilde{\epsilon} \epsilon) \hat{H}-\frac{i}{2}\left(\tilde{\epsilon} \gamma^{\mu \nu} \epsilon\right) F_{\mu \nu}(a)-\frac{1}{L} \hat{\sigma}+i\{c, \Psi\}, \\
Q c & =-\Lambda+\Lambda^{(0)}+\frac{i}{2}\{c, c\}, & Q \tilde{c}=b . &
\end{aligned}
$$

At this point it is worth to mention a point which will be important in the later analysis. In our $\xi$-gauge, we see from the ghost Lagrangian involving fields $(\widetilde{c}, b)$

$$
\mathcal{L}_{\widetilde{c}, b}=\operatorname{Tr}\left[i b \cosh ^{2} r \nabla_{\mu}\left(\frac{1}{\cosh ^{2} r} a^{\mu}\right)-\xi b^{2}+i \widetilde{c} \cosh ^{2} r \nabla_{\mu}\left(\frac{1}{\cosh ^{2} r} \partial^{\mu} c\right)+\xi \widetilde{c}\left(\mathcal{L}_{K} \widetilde{c}+i\left[\Lambda^{(0)}, \widetilde{c}\right]\right)\right],
$$

that if we choose

$$
\widetilde{c}=\frac{\vec{\mu}}{\cosh ^{2} r}, \quad b=\frac{\vec{\mu}^{\prime}}{\cosh ^{2} r},
$$

where $\vec{\mu}$ and $\vec{\mu}^{\prime}$ are gauge Lie algebra valued constant, then this mode decouples from the rest of the fields in the theory. The quadratic terms involving $b$ and $\widetilde{c}$ only gives a mass terms for this mode which is proportional to $\xi$. In fact, in $\xi=0$ limit these are zero modes. We will keep $\xi$ non zero for our convenience, however, we will subtract the contribution of this mode in the later calculation.

\subsection{Boundary conditions}

In this section we will discuss the boundary conditions on the fields present in the theory. This is essential when we define a quantum field theory on spaces with boundary. These boundary conditions set the value of the field at the boundary. In fact different boundary conditions define different quantum field theory. However, in the present case we are considering spaces which are of non compact type such as AdS. In this case the boundary conditions are much more reacher. AdS space being an open space, one needs to impose conditions on the asymptotic behaviour of fields. Typically, these asymptotic fall off conditions on fields are motivated by preserving certain aspect of the theory such as preserving certain symmetry, normalizability and the ones motivated from the AdS/CFT correspondence. Here, we follow normalizability as the criteria on the fall off conditions i.e. we require that fluctuations of all the fields present in the theory on AdS space should fall off asymptotically in a manner such that they are $L^{2}$-normalizable. Assuming this condition we find that for the bosonic fields in the vector multiplet, the fields should fall off asymptotically to satisfy

$$
e^{r / 2} a_{t} \rightarrow 0, \quad e^{r / 2} a_{r} \rightarrow 0, \quad e^{-r / 2} a_{\theta} \rightarrow 0, \quad e^{r / 2} \sigma \rightarrow 0 .
$$

Here $a_{\mu}$ and $\sigma$ are Lie algebra valued gauge field and scalar field, respectively. Similarly, requiring that the gaugino fields, $\lambda$ and $\widetilde{\lambda}$, are normalizable implies that

$$
\Psi_{t} \rightarrow 0, \quad \Psi_{r} \rightarrow 0, \quad e^{-r} \Psi_{\theta} \rightarrow 0, \quad \text { and } \quad \Psi \rightarrow 0 .
$$


Next, we want to define the boundary conditions on the ghost system. The ghost system consists of two grassmann odd scalar $c, \widetilde{c}$ and the Lagrange multiplier field $b$. The normalizable boundary condition on the Lagrange multiplier $b$ implies that the fluctuations should statisfy $e^{r / 2} b \rightarrow 0$. The boundary condition on the ghost field $c$ is chosen to be the same as in [17] i.e.

$$
c \rightarrow f(\theta)+e^{-r / 2} \widetilde{f}(\theta, \tau)+\ldots .
$$

This was motivated from the fact that $c$ is a gauge transformation parameter and we allow fluctuations of $c$ which does not change the boundary conditions on the gauge field. Once we have chosen the boundary conditions on the field $c$, the boundary condition on the ghost $\widetilde{c}$ is fixed by requiring that

$$
\int d^{2} x \sqrt{g} \widetilde{c} c<\infty
$$

This requires that the field $\widetilde{c}$ should satisfy $e^{r} \widetilde{c} \rightarrow 0$, i.e. it falls faster than $e^{-r}$. Later on, we will see that these boundary condition on $c$ and $\widetilde{c}$ are essential in order to construct their Green's function.

\subsection{Equations of motions and the Greens function}

As we explained earlier, the variation of the one loop determinant is given by the product of the variation of the differential operator and its Green's function. The differential operator appears at the quadratic order in the fluctuations in the $Q V$ action. The Green's function can be explicitly constructed out of the solutions of the equations of motions of the differential operator. However, in the supersymmetric case to evaluate the variation of the one loop determinant, we do not need the explicit form of these solutions rather only their asymptotic behaviour, which is a considerable simplifications. In this section, we will present these differential operator for both bosonic and fermionic fields and their Green's function. After this we will discuss the asymptotic behaviour of these differential operator which we will use to construct the asymptotic solutions. Furthermore, for the purposes of the presentation we will assume the Gauge group is $\mathrm{SU}(2)$, but near the end we will generalize the result to any arbitrary compact group.

Equations of motions. We begin with the bosonic fields. In the discussion below we will not care about the auxiliary field $H$, as its equation of motion is trivial and we assume that we have integrated it out in the path integral. The rest of the bosonic fields are the vector field $a_{\mu}$ and the scalar field $\sigma$ which are elements in the Lie algebra of $\mathrm{SU}(2)$. In the following discussion we will only consider the non-Cartan part of these fields. This is because the quadratic fluctuations containing the fields in the Cartan do not depend on $\alpha$ and thus, do not contribute to the variation in the one loop determinant. It is easy to see this in the bosonic action (3.8) (and similarly for fermionic action).

We first expand the fields in terms of Fourier modes and write the Lagrangian in terms of the following Fourier modes

$$
\begin{array}{ll}
a_{t}^{1}=\frac{1}{2} a_{t ; n, p}^{+}(r) e^{i(n t+p \theta)}+\frac{1}{2} a_{t ; n, p}^{-}(r) e^{-i(n t+p \theta)}, & a_{r}^{1}=\frac{i}{2} a_{r ; n, p}^{+}(r) e^{i(n t+p \theta)}-\frac{i}{2} a_{r ; n, p}^{-}(r) e^{-i(n t+p \theta)} \\
a_{\theta}^{1}=\frac{1}{2} a_{\theta ; n, p}^{+}(r) e^{i(n t+p \theta)}+\frac{1}{2} a_{\theta ; n, p}^{-}(r) e^{-i(n t+p \theta)}, & a_{t}^{2}=-\frac{i}{2} a_{t ; n, p}^{+}(r) e^{i(n t+p \theta)}+\frac{i}{2} a_{t ; n, p}^{-}(r) e^{-i(n t+p \theta)}
\end{array}
$$




$$
\begin{aligned}
a_{r}^{2} & =\frac{1}{2} a_{r ; n, p}^{+}(r) e^{i(n t+p \theta)}+\frac{1}{2} a_{r ; n, p}^{-}(r) e^{-i(n t+p \theta)}, & a_{\theta}^{2} & =-\frac{i}{2} a_{\theta ; n, p}^{+}(r) e^{i(n t+p \theta)}+\frac{i}{2} a_{\theta ; n, p}^{-}(r) e^{-i(n t+p \theta)} \\
\sigma^{1} & =\frac{1}{2} \sigma_{n, p}^{+} e^{i(n t+p \theta)}+\frac{1}{2} \sigma_{n, p}^{-} e^{-i(n t+p \theta)}, & \sigma^{2} & =\frac{1}{2 i} \sigma_{n, p}^{+} e^{i(n t+p \theta)}-\frac{1}{2 i} \sigma_{n, p}^{-} e^{-i(n t+p \theta)} .
\end{aligned}
$$

Here the labels on the fields are the usual labels of the Lie algebra $s u(2)$.

The equations of motion for the vector field and scalar field are obtained by varying the action with respect to $a_{\mu ; n, p}^{-}$and $\sigma_{n, p}^{-}$and can be written as

$$
\mathcal{M}_{b} E_{b ; n, p}^{+}(r) \equiv M_{2} \partial_{r}^{2} E_{b ; n, p}^{+}(r)+M_{1} \partial_{r} E_{b ; n, p}^{+}(r)+M_{0} E_{b ; n, p}^{+}(r)=0 .
$$

Here $M_{2,1,0}$ are $4 \times 4$ matrices whiose elements are functions of coordinate $r$. The explicit form of these matrices are given in appendix B. The column vector $E_{b ; n, p}^{+}(r)$ is given as

$$
E_{b ; n, p}^{+}(r)=\left(\begin{array}{c}
a_{t ; n, p}^{+}(r) \\
a_{r ; n, p}^{+}(r) \\
a_{\theta ; n, p}^{+}(r) \\
\sigma_{n, p}^{+}(r)
\end{array}\right) .
$$

Similar to bosonic case, we first expand the fermionic fields in terms of Fourier modes. We will not present here the Fourier expansion of the fermionic fields, but we follow closely to the bosonic case e.g.

$$
\begin{aligned}
\Psi_{t}^{1} & =\frac{1}{2} \Psi_{t ; n, p}^{+}(r) e^{i(n t+p \theta)}+\frac{1}{2} \Psi_{t ; n, p}^{-}(r) e^{-i(n t+p \theta)}, \\
\Psi_{r}^{1} & =\frac{i}{2} \Psi_{r ; n, p}^{+}(r) e^{i(n t+p \theta)}-\frac{i}{2} \Psi_{r ; n, p}^{-}(r) e^{-i(n t+p \theta)}, \\
\Psi_{\theta}^{1} & =\frac{1}{2} \Psi_{\theta ; n, p}^{+}(r) e^{i(n t+p \theta)}+\frac{1}{2} \Psi_{\theta ; n, p}^{-}(r) e^{-i(n t+p \theta)} .
\end{aligned}
$$

Then, the fermionic equations of motions are

$$
\mathcal{M}_{f} E_{f ; n, p}^{+}(r) \equiv M_{2 f} \partial_{r}^{2} E_{f ; n, p}^{+}(r)+M_{1 f} \partial_{r} E_{f ; n, p}^{+}(r)+M_{0 f} E_{f ; n, p}^{+}(r)=0 .
$$

Here $M_{2 f, 1 f, 0 f}$ are $6 \times 6$ matrices which are functions of coordinate $r$, and

$$
E_{f ; n, p}^{+}(r)=\left(\begin{array}{c}
\widetilde{\Psi}_{t ; n, p}^{+} \\
\widetilde{\Psi}_{r ; n, p}^{+} \\
\widetilde{\Psi}_{\theta ; n, p}^{+} \\
c_{n, p}^{+} \\
\widetilde{c}_{n, p}^{+} \\
\Psi_{n, p}^{+}
\end{array}\right)
$$

Here $\Psi_{\mu}=\widetilde{\Psi}_{\mu}-D_{\mu} c .^{7}$ The explicit form of these matrices are given in appendix B.

\footnotetext{
${ }^{7}$ Note that the change of the field variable does not involve $\alpha$. Therefore, one naively expects that the resultant Jacobian will not give any extra $\alpha$-dependent contribution. We have checked that this naive expectation is indeed correct.
} 
Greens function. The Green's function for the bosonic operator is a $4 \times 4$ matrix and satisfies the equation

$$
M_{2} \partial_{r}^{2} G_{b ; n, p}^{+}\left(r, r^{\prime}\right)+M_{1} \partial_{r} G_{b ; n, p}^{+}\left(r, r^{\prime}\right)+M_{0} G_{b ; n, p}^{+}\left(r, r^{\prime}\right)=\delta\left(r-r^{\prime}\right) .
$$

The explicit form of the matrices $M_{2,1,0}$ are given in the appendix B. One of the simplifications which occur for the choice of the gauge fixing Lagrangian (3.10) is that the equations of motion for the scalar decouples from the equations of motion of the vector field $a_{\mu}$. Thus, the bosonic Green's function is block diagonal and has the form (2.9) for $r<r^{\prime}$ and (2.10) for $r>r^{\prime}$, where in the present case, $G_{1}\left(r, r^{\prime}\right)$ (and $\left.G_{1}^{\prime}\left(r, r^{\prime}\right)\right)$ is $3 \times 3$ and $G_{2}\left(r, r^{\prime}\right)$ (and $\left.G_{2}^{\prime}\left(r, r^{\prime}\right)\right)$ is $1 \times 1$ matrix, respectively.

The continuity and discontinuity of the first derivative of the Green's function

$$
\left.G_{b ; n, p}^{+}\left(r, r^{\prime}\right)\right|_{r<r^{\prime}}-\left.G_{b ; n, p}^{+}\left(r, r^{\prime}\right)\right|_{r>r^{\prime}}=0
$$

and

$$
\left.\partial_{r} G_{b ; n, p}^{+}\left(r, r^{\prime}\right)\right|_{r<r^{\prime}}-\left.\partial_{r} G_{b ; n, p}^{+}\left(r, r^{\prime}\right)\right|_{r>r^{\prime}}=M_{2}^{-1}
$$

Similarly, the Green's function for the fermionic operator is a $6 \times 6$ matrix which satisfies the similar continuity and discontinuity relations as above.

\subsection{Boundary terms}

Next, we consider the variation of the one loop determinant with respect to the background parameter $\alpha$. The variation is

$$
\frac{\delta}{\delta \alpha} \ln Z_{1 \text {-loop }}(\alpha)=\operatorname{Tr}\left[G_{F} \frac{\delta}{\delta \alpha} \mathcal{D}_{F}(\alpha)\right]-\frac{1}{2} \operatorname{Tr}\left[G_{B} \frac{\delta}{\delta \alpha} \mathcal{D}_{B}(\alpha)\right],
$$

where $\mathcal{D}_{F}(\alpha)$ and $\mathcal{D}_{B}(\alpha)$ are fermionic and bosonic kinetic operator, respectively. Following the discussion presented in the section 2.2 we find that in the supersymmetric case the variation is a total derivative and is given as

$$
\frac{\delta}{\delta \alpha} \ln Z_{1-\operatorname{loop}}(\alpha)=-\left.\delta_{\alpha}\left(\ln Q^{2}\right) \operatorname{tr} B_{1} D_{0}^{-1} C G_{1}(r, r)\right|_{r=0} ^{r=\infty},
$$

where $G_{1}\left(r, r^{\prime}\right)$ is the bosonic Green's function which is constructed out of the solutions of the equations of motions for the vector field and

$$
B=B_{1} \frac{\partial}{\partial r}+B_{0}, \quad C=C_{1} \frac{\partial}{\partial r}+C_{0} .
$$

The explicit forms of these matrices are

$$
B_{1}=\left(\begin{array}{cc}
0 & -\frac{i L}{2} \tanh ^{2} r \\
-\frac{i}{2} \sinh r & 0 \\
0 & \frac{i}{2} \operatorname{sech}^{2} r
\end{array}\right), \quad B_{0}=\left(\begin{array}{cc}
\frac{i}{2} L^{2} n \sinh r & -i L \operatorname{sech}^{2} r \tanh r \\
-i \sinh r \tanh r \frac{i}{2 \cosh ^{2} r}\left(-p+L n \sinh ^{2} r\right) \\
\frac{i}{2} \frac{p}{\sinh r} & -i \operatorname{sech}^{2} r \tanh r
\end{array}\right),
$$


and

$$
C_{1}=\left(\begin{array}{ccc}
0 & \frac{i}{2} \sinh r & 0 \\
\frac{i}{2} L \tanh ^{2} r & 0 & -\frac{i}{2} \operatorname{sech}^{2} r
\end{array}\right), C_{0}=\left(\begin{array}{ccc}
\frac{i}{2} L^{2} n \sinh r & \frac{i}{4 \cosh r}(3-\cosh 2 r) & \frac{i p}{2 \sinh r} \\
0 & \frac{i}{2}\left(L n \sinh ^{2} r-p\right) \operatorname{sech}^{2} r & 0
\end{array}\right) .
$$

It is not very hard to see that the differential operator $B$ and $C$ are adjoint to each other, i.e. $B=C^{\dagger}$. The operator $D$ is algebraic (not a differential operator) and is given by

$$
D=\left(\begin{array}{cc}
i L \xi(p+L(n-\alpha)) \sinh r & 0 \\
0 & -\frac{i}{2} L(p+L(n-\alpha)) \operatorname{sech} r \tanh r
\end{array}\right) .
$$

Note that the matrix operator $B$ (and $C$ ) are independent of $\alpha$. Furthermore, the $\alpha$ dependence in the matrix $D$ is of the form $Q^{2}$ and therefore, the matrix $D$ can be written as $Q^{2} D_{0}$, where $D_{0}$ is independent of $\alpha$. Thus, it justifies the form of the variation (3.31) where $\delta_{\alpha}$ acts only on $D$.

It is important to emphasize here that the variation being a total derivative (3.31) depends on the boundary conditions. In fact, the derivation assumes that the fluctuations of fermionic and bosonic fields obey boundary conditions which are consistent with susy. In other words, the fermionic kinetic operator is related to bosonic kinetic operator by a similarity transformations ${ }^{8}$ and therefore, the fermionic and bosonic Green's functions are related by similarity transformations. We will show below that this is true if $L^{2}>\frac{3}{4}$. When $L^{2}<\frac{3}{4}$, the variation of the one loop determinant will not just be a boundary term but will also contain bulk terms [18].

\subsection{Evaluating boundary terms}

Next, we evaluate the boundary term (3.31). To evaluate this we just need to determine the action of the first order differential operator $B_{1} D_{0}^{-1} C$ on the Green's function $G_{1}\left(r, r^{\prime}\right)$ and their asymptotic behaviour. Interestingly, we do not need to know the complete details of the Green's function except it's asymptotic behaviour. As we will see below, this greatly simplifies the computations. The Green's function is constructed from the solutions of the equations of motions and we will only need to know the asymptotic behaviour of the solutions.

Now, the Green's function $G_{1}\left(r, r^{\prime}\right)$ satisfies

$$
\left.\mathcal{M}_{b}\right|_{X_{0}} G_{1}\left(r, r^{\prime}\right) \equiv\left(m_{b 2} \partial_{r}^{2}+m_{b 1} \partial_{r}+m_{b 0}\right) G_{1}\left(r, r^{\prime}\right)=\delta\left(r, r^{\prime}\right) \text {. }
$$

Here $m_{b 2,1,0}$ are $3 \times 3$ matrices acting on $X_{0}$ only (which are component of the vector fields). The differential operator $\left.\mathcal{M}_{b}\right|_{X_{0}}$ is obtained by projecting the operator $\mathcal{M}_{b}$ to the vector space $X_{0}$

$$
\left.\mathcal{M}_{b}\right|_{X_{0}}=\mathcal{P} \mathcal{M}_{b} \mathcal{P}^{T}
$$

\footnotetext{
${ }^{8}$ One can show that fermionic kinetic operator is $M_{f}=\left(E^{\dagger}\right)^{-1}\left(\begin{array}{ccc}\gamma_{1} A_{1}^{b} & 0 & 0 \\ 0 & \gamma_{2} A_{2}^{b} & 0 \\ 0 & 0 & D\end{array}\right) E^{-1}$, where $E$ is a $((2 k+2) \times(2 k+2))$ matrix first order differential operator and $\gamma_{1}=\frac{1}{Q^{2}}=\frac{1}{\gamma_{2}}$.
} 
where

$$
\mathcal{P}=\left(\begin{array}{llll}
1 & 0 & 0 & 0 \\
0 & 1 & 0 & 0 \\
0 & 0 & 1 & 0
\end{array}\right)
$$

In the discussion presented in the section 2.3, it turned out to be useful to split the vector space into a rank 1 and rank 2 subspaces. The rank 2 subspace was defined to be the one whose elements are orthogonal to the vector $K$ and the rank 1 whose elements orthogonal to $C_{1}$. Following the same spirit, we split the vector space $X_{0}$ which we denote by $V$ into $V_{1}$ and $V_{2}$. In the present case, the dimension of the vector space $V, V_{1}$ and $V_{2}$ are 3,2 and 1 , respectively. To define the vector space $V_{1}$ we need the vector $K$ which is given as (see the appendix for more details)

$$
K=\frac{1}{p+L(n-\alpha)}\left(\begin{array}{lll}
L & 0 & 1
\end{array}\right)
$$

A typical vector in $V_{1}$ has the form

$$
v_{1}=\left(\begin{array}{c}
-\frac{x_{1}}{L} \\
x_{2} \\
x_{1}
\end{array}\right), \quad x_{1,2} \in \mathbb{R}
$$

and that belonging to the vector space $V_{2}$ has the form

$$
v_{2}=x\left(\begin{array}{c}
1 \\
0 \\
L \sinh ^{2} r
\end{array}\right), \quad x \in \mathbb{R}
$$

In order to simplify the computations, we change the basis of the vector space $V$ such that the first two non zero component belongs to the vector space $V_{1}$ and the 3rd non zero component belongs to the vector space $V_{2}$. That is given a vector $v \in V$, we define a vector $\widetilde{v}$ as $v=J \widetilde{v}$ such that for $\widetilde{v}=\left(\begin{array}{c}c_{1} \\ c_{2} \\ 0\end{array}\right)$, for $c_{1,2} \in \mathbb{R}$, the corresponding $v \in V_{1}$ and for $\widetilde{v}=\left(\begin{array}{c}0 \\ 0 \\ c_{3}\end{array}\right)$, for $c_{3} \in \mathbb{R}$, the corresponding $v \in V_{2}$. It turns out that there is no unique choice of $J$ (different $J$ 's are related to each other by rotation in $V_{1}$ space) and one convenient choice is

$$
J=\left(\begin{array}{ccc}
0 & \frac{2}{L} \tanh r & \frac{2}{L} \operatorname{sech} r \\
2 & 0 & 0 \\
0 & -2 \tanh r & 2 \sinh r \tanh r
\end{array}\right)
$$

Subsequently, the corresponding matrix operator acting on the elements of the vector space $\widetilde{V}$ is related to the original operator by similarity transformations as

$$
m_{b 2 p, b 1 p, b 0 p}=J^{T} m_{b 2, b 1, b 0} J .
$$


Asymptotic behaviour of differential operator. As we found earlier in (3.31) that to evaluate the variation of the one loop determinant, we just need to know the asymptotic behaviour of the Green's function. Now, the Green's functions are constructed out of the solutions of the equations of motion. Thus for our purposes to evaluate the boundary terms (3.31), the global form of the solutions are not necessary rather its asymptotic form will suffice. Furthermore, we argued there that the contributions to the boundary terms only come from the space of the solutions belonging to the vector space $V_{1}$. Thus we need to construct the Green's function restricted to the vector space $V_{1}$ i.e.

$$
\left.G_{1}\left(r, r^{\prime}\right)\right|_{V_{1}}=P G_{1}\left(r, r^{\prime}\right) P^{T}
$$

where the projection operator is

$$
P=\left(\begin{array}{lll}
1 & 0 & 0 \\
0 & 1 & 0
\end{array}\right) .
$$

To obtain the asymptotic form of the solutions, we need to analyse the asymptotic behaviour of the kinetic operator near $r=0$ and $r=\infty$. Near $r \rightarrow 0$, the leading contributions to matrix coefficients of the 2nd order differential operator are

$$
\begin{aligned}
& \lim _{r \rightarrow 0} m_{b 2 p}=\frac{r}{L^{2}}\left(\begin{array}{ccc}
\frac{1}{\xi} & 0 & 0 \\
0 & -2 & 0 \\
0 & 0 & -2
\end{array}\right)+\mathcal{O}\left(r^{3}\right), \lim _{r \rightarrow 0} m_{b 1 p}=\frac{1}{\xi L^{2}}\left(\begin{array}{ccc}
1 & -p(1+2 \xi) & 0 \\
p(1+2 \xi) & -2 \xi & 0 \\
0 & 0 & -2 \xi
\end{array}\right)+\mathcal{O}(r) \\
& \lim _{r \rightarrow 0} m_{b 0 p}=\frac{1}{L^{2} r \xi}\left(\begin{array}{ccc}
2 p^{2} \xi-1 & p(1-2 \xi) & 0 \\
p(1-2 \xi) & 2 \xi-p^{2} & 0 \\
0 & 0 & 2 p^{2} \xi
\end{array}\right)+\mathcal{O}(1)
\end{aligned}
$$

On the other hand near $r \rightarrow \infty$, the leading behaviour of the differential operator is

$$
\begin{aligned}
& \lim _{r \rightarrow \infty} m_{b 2 p}=\frac{1}{u L^{2}}\left(\begin{array}{ccc}
\frac{1}{2 \xi} & 0 & 0 \\
0 & -1 & 0 \\
0 & 0 & -1
\end{array}\right)+\mathcal{O}(1), \lim _{r \rightarrow \infty} m_{b 1 p}=\frac{1}{u L^{2}}\left(\begin{array}{ccc}
\frac{1}{2 \xi} & \frac{L n(1+2 \xi)}{2 \xi} & 0 \\
-\frac{L n(1+2 \xi)}{2 \xi} & -1 & 0 \\
0 & 0 & -1
\end{array}\right)+\mathcal{O}(1) \\
& \lim _{r \rightarrow \infty} m_{b 0 p}=\frac{1}{u L}\left(\begin{array}{ccc}
L n^{2}-\frac{1}{L \xi} & \frac{n}{\xi} & 0 \\
\frac{n(1-2 \xi)}{2 \xi} & -\frac{L n^{2}}{2 \xi} & 0 \\
0 & 0 & L n^{2}
\end{array}\right)+\mathcal{O}(1)
\end{aligned}
$$

Here $u=e^{-r}$.

It is important to observe that the second order differential operator $\left.J^{T} B D^{-1} C J\right|_{V_{1}}$ has the same asymptotic behaviour as the differential operator 
Solutions near $\boldsymbol{r} \rightarrow \mathbf{0}$. The asymptotic behaviour of the solution near $r \rightarrow 0$ is controlled by the integer $p$ and is independent of $n$. Solving the equations of motion near $r \rightarrow 0$ we find that, for $p>0$, there are 3 smooth solutions which are

$$
\begin{aligned}
& s_{1 p}(r)=r^{p-1}\left(\begin{array}{l}
1 \\
1 \\
0
\end{array}\right) \in V_{1}, \quad s_{2 p}(r)=r^{p+1}\left(\begin{array}{c}
\frac{p(1+2 \xi)+4 \xi}{2+p(1+2 \xi)} \\
1 \\
0
\end{array}\right) \in V_{1}, \\
& s_{3 p}(r)=r^{p}\left(\begin{array}{l}
0 \\
0 \\
1
\end{array}\right) \in V_{2},
\end{aligned}
$$

and 3 singular solutions which are

$$
\begin{aligned}
& s_{4 p}(r)=r^{-p-1}\left(\begin{array}{c}
1 \\
-1 \\
0
\end{array}\right) \in V_{1}, \quad s_{5 p}(r)=r^{-p+1}\left(\begin{array}{c}
1 \\
-\frac{p(1+2 \xi)-2}{p(1+2 \xi)-4 \xi} \\
0
\end{array}\right) \in V_{1}, \\
& s_{6 p}(r)=r^{-p}\left(\begin{array}{l}
0 \\
0 \\
1
\end{array}\right) \in V_{2} .
\end{aligned}
$$

For $p<0$, the solutions $s_{4 p, 5 p, 6 p}$ are smooth and $s_{1 p, 2 p, 3 p}$ are singular.

For the case of $p=0$, we see that $s_{3}(r)$ and $s_{6}(r)$ are degenerate. Solving next to leading order we find two linearly independent solutions and are given by

$$
s_{30}(r)=\left(\begin{array}{l}
0 \\
0 \\
1
\end{array}\right) \in V_{2}, \quad s_{60}(r)=\ln r\left(\begin{array}{l}
0 \\
0 \\
1
\end{array}\right) \in V_{2} .
$$

Thus, for $p=0$, the solutions which are smooth are $s_{20,30,50}$ whereas $s_{10,40,60}$ are singular near $r \rightarrow 0$, where $s_{20,50}$ and $s_{10,40}$ are obtained by putting $p=0$ in $s_{2 p, 5 p}$ and $s_{1 p, 4 p}$, respectively.

Since $\widetilde{\Psi}_{\mu}$ satisfies the same equations of motion as the vector field, therefore, the smooth solutions for the vector field are also smooth for $\widetilde{\Psi}_{\mu}$. Near $r \rightarrow 0$ behaviour of the solution for $(\widetilde{c}, \Psi)$ is obtained from $\widetilde{\Psi}_{\mu}$ as

$$
\left(\begin{array}{c}
\widetilde{c}_{n, p}^{+} \\
\Psi_{n, p}^{+}
\end{array}\right)=-D^{-1} C\left(\begin{array}{c}
\widetilde{\Psi}_{t ; n, p}^{+} \\
\widetilde{\Psi}_{r ; n, p}^{+} \\
\widetilde{\Psi}_{\theta ; n, p}^{+}
\end{array}\right) .
$$

Using the solutions given in (3.48) and (3.49) for $p>0$, we find that $s_{1 p}(r), s_{2 p}(r)$ and $s_{3 p}(r)$ also give rise smooth solutions for $\widetilde{c}$ and $\Psi$. For example when $s_{1 p}(r), s_{2 p}(r)$ and 
$s_{3 p}(r)$ are acted upon by the differential operator $-D^{-1} C$, we get near $r \rightarrow 0$

$$
-D^{-1} C s_{1 p}(r) \sim\left(\begin{array}{c}
c_{1} \\
c_{2}
\end{array}\right) r^{p}, \quad-D^{-1} C s_{2 p}(r) \sim c_{3}\left(\begin{array}{c}
-1 \\
1
\end{array}\right) r^{p}, \quad-D^{-1} C s_{3 p}(r) \sim\left(\begin{array}{c}
c_{4} \\
c_{5}
\end{array}\right) r^{p} .
$$

Here $c_{i}$ 's are constants. Thus, for $p>0$ above are smooth solutions for $\widetilde{c}$ and $\Psi$. Similarly, it is not difficult to see that $s_{4 p, 5 p, 6 p}$ do not give smooth solution near $r \rightarrow 0$. For $p<0$, the smooth solutions for $\widetilde{c}$ and $\Psi$ are obtained from $s_{4 p, 5 p, 6 p}$ whereas $s_{1 p, 2 p, 3 p}$ give rise singular solutions. For $p=0, s_{i 0}$ for $i=1, \ldots 5$ give smooth solutions for $\widetilde{c}$ and $\Psi$. Thus, for the fermionic system $\left(\widetilde{\Psi}_{\mu}, \widetilde{c}, \Psi\right)$, for $p=0$, the smooth solutions are $s_{20,30,50}$ whereas $s_{10,40,60}$ are singular.

Solutions near $r \rightarrow \infty$. Next, we determine the asymptotic behaviour of the solutions near $r \rightarrow \infty$. The asymptotic behaviour of the solution near $r \rightarrow \infty$ is controlled by the integer $n$ and is independent of $p$. We find that for $L^{2} n^{2}>\frac{3}{4}$, following are the asymptotic behaviour of normalizable solutions (normalizability conditions for the component of gauge field are given in (3.17))

$$
\begin{gathered}
\widetilde{s}_{1 n}=\left(\begin{array}{c}
c(n) \\
1 \\
0
\end{array}\right) e^{-\frac{r}{2}\left(3+\sqrt{1+4 L^{2} n^{2}}\right)} \in V_{1}, \quad \widetilde{s}_{2 n}=\left(\begin{array}{c}
\widetilde{c}(n) \\
1 \\
0
\end{array}\right) e^{-\frac{r}{2}\left(-1+\sqrt{1+4 L^{2} n^{2}}\right)} \in V_{1}, \\
\widetilde{s}_{3 n}=\left(\begin{array}{l}
0 \\
0 \\
1
\end{array}\right) e^{-\frac{r}{2}\left(1+\sqrt{1+4 L^{2} n^{2}}\right)} \in V_{2} . \\
\text { Here } c(n)=\frac{L n\left(-1+6 \xi+\sqrt{1+4 L^{2} n^{2}}(1+2 \xi)\right)}{2\left(-1+\sqrt{1+4 L^{2} n^{2}}+L^{2} n^{2}(1+2 \xi)\right)} \text { and } \widetilde{c}(n)=-\frac{L n\left(-5-2 \xi+\sqrt{1+4 L^{2} n^{2}}(1+2 \xi)\right)}{2\left(1+\sqrt{1+4 L^{2} n^{2}}-L^{2} n^{2}(1+2 \xi)\right)}
\end{gathered}
$$

The asymptotic behaviour of solutions which are not normalizable are

$$
\begin{aligned}
& \widetilde{s}_{4 n}=\left(\begin{array}{c}
c_{1}(n) \\
1 \\
0
\end{array}\right) e^{-\frac{r}{2}\left(-1-\sqrt{1+4 L^{2} n^{2}}\right)} \in V_{1}, \quad \widetilde{s}_{5 n}=\left(\begin{array}{c}
\widetilde{c}_{1}(n) \\
1 \\
0
\end{array}\right) e^{-\frac{r}{2}\left(3-\sqrt{1+4 L^{2} n^{2}}\right)} \in V_{1}, \\
& \widetilde{s}_{6 n}=\left(\begin{array}{l}
0 \\
0 \\
1
\end{array}\right) e^{-\frac{r}{2}\left(1-\sqrt{1+4 L^{2} n^{2}}\right)} \in V_{2} .
\end{aligned}
$$

Here $c_{1}(n)=-\frac{L n\left(5+2 \xi+\sqrt{1+4 L^{2} n^{2}}(1+2 \xi)\right)}{2\left(-1+\sqrt{1+4 L^{2} n^{2}}+L^{2} n^{2}(1+2 \xi)\right)}$ and $\widetilde{c}_{1}(n)=\frac{\operatorname{Ln}\left(1-6 \xi+\sqrt{1+4 L^{2} n^{2}}(1+2 \xi)\right)}{2\left(1+\sqrt{1+4 L^{2} n^{2}}-L^{2} n^{2}(1+2 \xi)\right)}$.

However, for $0<L^{2} n^{2}<\frac{3}{4}$, we find that the normalizable solutions are $\widetilde{s}_{1 n}(r), \widetilde{s}_{3 n}(r)$ and $\widetilde{s}_{5 n}(r)$ and non normalizable solutions are $\widetilde{s}_{2 n}(r), \widetilde{s}_{4 n}(r)$ and $\widetilde{s}_{6 n}(r)$. The solution 
with $n=0$ will play an important role for later analysis, we present here their explicit form. For $n=0$, the asymptotic behaviour of normalizable solutions are

$$
\begin{aligned}
& \widetilde{s}_{10}(r)=\left(\begin{array}{l}
1 \\
0 \\
0
\end{array}\right) e^{-2 r} \in V_{1}, \quad \widetilde{s}_{30}(r)=\left(\begin{array}{l}
0 \\
0 \\
1
\end{array}\right) e^{-r} \in V_{2}, \\
& \widetilde{s}_{50}(r)=\left(\begin{array}{l}
0 \\
1 \\
0
\end{array}\right) e^{-r} \in V_{1} .
\end{aligned}
$$

and the asymptotic behaviour of the non normalizable solutions are

$$
\begin{aligned}
& \widetilde{s}_{20}(r)=\left(\begin{array}{l}
0 \\
1 \\
0
\end{array}\right) \in V_{1}, \quad \widetilde{s}_{40}(r)=\left(\begin{array}{l}
1 \\
0 \\
0
\end{array}\right) e^{r} \in V_{1}, \\
& \widetilde{s}_{60}(r)=\left(\begin{array}{l}
0 \\
0 \\
1
\end{array}\right) \in V_{2} .
\end{aligned}
$$

Now, we discuss asymptotic behaviour of solutions belonging to fermionic system $\left(\widetilde{\Psi}_{\mu}, c\right.$, $\widetilde{c}, \Psi)$. Since $\widetilde{\Psi}_{\mu}$ satisfies the same equation of motion as the vector field, the solutions of vector field are also solutions for the fermion $\widetilde{\Psi}_{\mu}$. However, fields $\left(\widetilde{\Psi}_{\mu}, c, \widetilde{c}, \Psi\right)$ have different normalizabilty conditions, see (3.18) and (3.19), and therefore, we need to reanalyse which of the solutions among the set of solutions obtained above are normalizable and non normalizable, respectively for fermions. Before going to analyse the above solutions for fermions, it is important to mention a few comments about the equation of motion satisfied by $c$. From susy algebra (3.14), we see that if we replace $c$ by $\cosh r \hat{c}-\frac{1}{Q^{2}} K^{\mu} \widetilde{\Psi}_{\mu}$, then $\hat{c}$ satisfies the same equation as $\sigma$. Solving the equation of motion for $\sigma$ we find that there are 2 solutions with asymptotic behaviour near $r=\infty$

$$
\sigma_{n, p} \sim A_{1} e^{-\frac{r}{2}\left(1-\sqrt{1+4 L^{2} n^{2}}\right)}+A_{2} e^{-\frac{r}{2}\left(1+\sqrt{1+4 L^{2} n^{2}}\right)} .
$$

The normalizablity condition on $\sigma$ requires us to choose the second solution. Since $\hat{c}_{n, p}$ satisfies the same equation as $\sigma_{n, p}$, we have the same asymptotic behaviour for $\hat{c}_{n, p}$. Thus, it is easy to see that for the ghost $c_{n, p}$, it is only the 2 nd solution (labelled by $A_{2}$ ) which will give admissible asymptotic behaviour. Furthermore, given the asymptotic behaviour of the solutions $\widetilde{\Psi}_{\mu}$, the asymptotic behaviour of $(\widetilde{c}, \Psi)$ is obtained by using (3.51). Now, we will tabulate these solutions in table 1 indicating whether they are normalizable (marked by $\checkmark$ ) or nonnormalizable (marked by X). Looking at the table, we see that the solutions for bosonic and fermionic fields are consistent with supersymmetry only for $L^{2} n^{2}>\frac{3}{4}$. For the range $0<L^{2} n^{2}<\frac{3}{4}$, we find that $\widetilde{s}_{1 n}(r), \widetilde{s}_{3 n}(r)$ and $\widetilde{s}_{5 n}(r)$ are normalizable for the gauge field whereas $\widetilde{s}_{1 n}(r), \widetilde{s}_{2 n}(r)$ and $\widetilde{s}_{3 n}(r)$ are normalizable for fermionic fields. Thus, there is a mismatch of the space of allowed solutions for fermionic and bosonic fields. In this situation, the Green's function for the bosonic field is not related to that of the fermionic 


\begin{tabular}{|c|c|c|c|c|}
\hline Solutions & $\widetilde{\Psi}_{\mu}$ & $c=\cosh r \hat{c}-\frac{1}{Q^{2}} K^{\mu} \widetilde{\Psi}_{\mu}$ & $\widetilde{c}$ & $\Psi$ \\
\hline$\widetilde{s}_{1 n}(r)$ & $\checkmark \forall n \neq 0$ & $\checkmark \forall n \neq 0$ & $\checkmark \forall n \neq 0$ & $\checkmark \forall n \neq 0$ \\
\hline$\widetilde{s}_{2 n}(r)$ & $\checkmark \forall n \neq 0$ & $\checkmark \forall n \neq 0$ & $\checkmark \forall n \neq 0$ & $\checkmark \forall n \neq 0$ \\
\hline$\widetilde{s}_{3 n}(r)$ & $\checkmark \forall n \neq 0$ & $\checkmark \forall n \neq 0$ & $\checkmark \forall n \neq 0$ & $\checkmark \forall n \neq 0$ \\
\hline$\widetilde{s}_{4 n}(r)$ & $\mathrm{X} \forall n \neq 0$ & $\mathrm{X} \forall n \neq 0$ & $\mathrm{X} \forall n \neq 0$ & $\mathbf{X} \forall n \neq 0$ \\
\hline$\widetilde{s}_{5 n}(r)$ & $\checkmark 0<\forall L^{2} n^{2}<2$ & $\checkmark 0<\forall L^{2} n^{2} \leq 2$ & $\mathrm{X} \forall n \neq 0$ & $\mathbf{X} \forall n \neq 0$ \\
\hline$\widetilde{s}_{6 n}(r)$ & $\mathrm{X} \forall n \neq 0$ & $\mathrm{X} \forall n \neq 0$ & $\mathrm{X} \forall n \neq 0$ & $\mathbf{X} \forall n \neq 0$ \\
\hline
\end{tabular}

Table 1. Summary of acceptable and non acceptable solutions for fermionic fields.

field and, therefore, for the modes lying in the interval, $0<L^{2} n^{2}<\frac{3}{4}$, the variation of the one loop determinant will not be just a boundary term but will also include bulk terms. To determine the explicit expression for the bulk term we need to know the global form of the solutions and not just the asymptotic behaviour. This is a much more harder problem in the present case where we do not have the global form of the solution. To avoid this, we assume that $L^{2}>\frac{3}{4}$. With this there are no modes lying in the interval $0<L^{2} n^{2}<\frac{3}{4}$.

Now, we will discuss the case of $n=0$. In this case the analysis is slightly subtle and needs a separate discussion.

Case: $\boldsymbol{n}=\mathbf{0}$. The acceptable solutions for the bosonic fields are given in (3.55). Next we need to analyze whether these solutions give rise acceptable solutions to fermionic fields. In this case it turns out that $\widetilde{s}_{10}$ and $\widetilde{s}_{30}$ give rise normalizable solutions, while $\widetilde{s}_{40}$ and $\widetilde{s}_{60}$ give rise nonnormalizable solutions to the fermionic fields. The asymptotic behaviour of the solutions $\widetilde{s}_{50}$ and $\widetilde{s}_{20}$ are subtle for fermionic fields. For these solutions, we find the following: for the solutions $\widetilde{s}_{50}$, the asymptotic behaviour of fermionic fields as $r \rightarrow \infty$ are

$$
\widetilde{s}_{50}: \quad \widetilde{\Psi}_{t} \sim e^{-r}, \widetilde{\Psi}_{r} \sim 0, \widetilde{\Psi}_{\theta} \sim e^{-r}, \quad \widetilde{c} \sim e^{-3 r}, \Psi \sim \mathcal{O}(1), c \sim \mathcal{O}(1),
$$

whereas for the solutions $\widetilde{s}_{20}$, the asymptotic behaviour of fermionic fields as $r \rightarrow \infty$ are

$$
\widetilde{s}_{20}: \quad \widetilde{\Psi}_{t} \sim \mathcal{O}(1), \widetilde{\Psi}_{r} \sim 0, \widetilde{\Psi}_{\theta} \sim \mathcal{O}(1), \quad \widetilde{c} \sim e^{-r}, \Psi \sim e^{-r}, c \sim \mathcal{O}(1) .
$$

Comparing these asymptotic behaviour with the boundary conditions (3.18) and the boundary condition on $\widetilde{c}$, one would naively declare both the above solutions to be non normalizable. But this would amount to non existence of Green's function. The requirement of the existence of the Green's function forces us to declare one of these solution to be normalizable and other to be nonnormalizable. Thus, for the case of $n=0$ and $p \neq 0$ we have two choices:

1) We declare that $\widetilde{s}_{50}$ is normalizable and $\widetilde{s}_{20}$ is nonnormalizable which would correspond to preserving supersymmetry, or

2) We declare $\widetilde{s}_{20}$ to be normalizable and $\widetilde{s}_{50}$ nonnormalizable, then this would break the supersymmetry. 
Making either of the choices requires to modify (although minimally) the boundary conditions we started with. Since we are only interested in the boundary terms, which is the case when the allowed modes are also consistent with supersymmetry, we choose the option 1 . It is definitely worth to try with option 2 , but in this case we also need to calculate the bulk term (because for this choice we do not have supersymmetric cancellation) which is beyond the scope of the present paper. To allow the option 1, we modify the boundary conditions (3.18) which would amount to following asymptotic behaviour

$$
\Psi_{t} \rightarrow 0, \quad \Psi_{r} \rightarrow 0, \quad e^{-r} \Psi_{\theta} \rightarrow 0, \quad \text { and } \quad \Psi \rightarrow \mathcal{O}(1) .
$$

Note that this choice does not change the analysis presented above for the case $n \neq 0$.

\subsection{Variation of the one loop partition function}

As it was shown in the section 2.3 that to determine the boundary contribution we just need to know the dimension of the kernel of the operator $\left.C\right|_{V_{1}}$ i.e. $\ell$ and $\ell^{\prime}$ near $r \rightarrow 0$ and $r \rightarrow \infty$, respectively. Theses dimensions of the kernel of the operator $\left.C\right|_{V_{1}}$ depends on the value of $(n, p)$. We split the evaluation of the boundary term in following 4 different cases:

Case: $\boldsymbol{p} \neq \mathbf{0}, \boldsymbol{n} \neq \mathbf{0}$. We start with the computation of the boundary term near $r \rightarrow 0$. As we found earlier, the asymptotic behaviour of the solutions in this limit depends only on the value of $p$ and are independent of $n$. The solutions which are admissible near $r \rightarrow 0$ for $p>0$ and $p<0$ are $s_{1 p}(r)$ and $s_{2 p}(r)$, and $s_{4 p}(r)$ and $s_{5 p}(r)$, respectively. However, it is only $s_{1 p}(r)\left(s_{4 p}(r)\right)$ belongs to the kernel of $\left.C\right|_{V_{1}}$ for $p>0(p<0)$ i.e.

$$
\left.\lim _{r \rightarrow 0} C\right|_{V_{1}} s_{1 p}(r)=0, \quad \text { for } p>0,
$$

and

$$
\left.\lim _{r \rightarrow 0} C\right|_{V_{1}} s_{4 p}(r)=0, \quad \text { for } p<0 .
$$

Thus the dimension of the kernel, $\ell$, for $p \neq 0$ is 1 .

Near $r \rightarrow \infty$, the admissible solutions are $s_{1 n}(r)$ and $s_{2 n}(r)$. However, the solution which belongs to the kernel of $\left.C\right|_{V_{1}}$ is $s_{2 n}(r)$ i.e.

$$
\left.\lim _{r \rightarrow \infty} C\right|_{V_{1}} s_{2 n}(r)=0 .
$$

Thus, we have $\ell^{\prime}=1$. Therefore, the boundary contribution for the case $n \neq 0$ and $p \neq 0$ is

$$
\mathrm{BT}=\left(k-\ell-\ell^{\prime}\right)=2-1-1=0 .
$$

Case: $\boldsymbol{p} \neq \mathbf{0}, \boldsymbol{n}=\mathbf{0}$. Since the asymptotic behaviour near $r \rightarrow 0$ for $p \neq 0$ does not depend on $n$, the dimension of the kernel, $\ell$, remains same as before and is equal to 1 . However, for $n=0$ we find that there no normalizable modes in (3.55) which belongs to the kernel of $\left.C\right|_{V_{1}}$. Thus in this case we have $\ell^{\prime}=0$. Therefore, the boundary contribution for the case $n=0$ and $p \neq 0$ is

$$
\mathrm{BT}=\left(k-\ell-\ell^{\prime}\right)=2-1=1 .
$$


Case: $\boldsymbol{n} \neq \mathbf{0}, \boldsymbol{p}=\mathbf{0}$. Since the asymptotic behaviour near $r \rightarrow \infty$ for $n \neq 0$ does not depend on $p$, the dimension of the kernel, $\ell^{\prime}$, remains same as before and is equal to 1 . However, for $p=0$ we find that there no smooth modes which belongs to the kernel of $\left.C\right|_{V_{1}}$. Thus in this case we have $\ell=0$. Therefore, the boundary contribution for the case $p=0$ and $n \neq 0$ is

$$
\mathrm{BT}=\left(k-\ell-\ell^{\prime}\right)=2-1=1 .
$$

Case: $\boldsymbol{n}=\boldsymbol{p}=\mathbf{0}$. Following the discussion of $n=0, p \neq 0$ and $p=0, n \neq 0$ cases we find that dimensions of the kernel of $\left.C\right|_{V_{1}}$ in the case of $n=p=0$ are $\ell=\ell^{\prime}=0$. Thus, its contribution to the boundary term is

$$
\mathrm{BT}=2 .
$$

It was observed in [17] that this contribution to the index comes precisely from the zero modes of the ghost fields which were given by globally constants mode for ghost $c$ and anti ghost $\widetilde{c}$. Since the determinant are computed over non zero modes, we did not include the contribution of these zero modes.

We also observe this fact in our present computation. First zero mode corresponds scalar fluctuations parallel to the localization background i.e.

$$
\hat{\sigma}=\frac{\vec{A}}{\cosh r}, \quad \vec{A}=\text { constant Lie algebra element } .
$$

The supersymmetric partner of the above zero mode is the constant ghost mode $c=\vec{A}$ (it can be seen following (3.12)).

As we discussed near (3.16), the second zero mode corresponds to

$$
\widetilde{c}=\frac{\vec{\mu}}{\cosh ^{2} r}, \quad b=\frac{\vec{\mu}_{Q}}{\cosh ^{2} r}, \quad \text { where } Q \vec{\mu}_{Q}=i\left[\Lambda^{(0)}, \vec{\mu}\right]
$$

where $\vec{\mu}$ and $\vec{\mu}_{Q}$ are Grassmann odd and even constant Lie algebra element, respectively. The ghost Lagrangian involves mass like terms

$$
\frac{\xi}{\cosh ^{2} r}\left(\operatorname{tr} \vec{\mu}_{Q}^{2}+2 \sum_{\vec{\rho}>0} \rho \cdot \alpha \mu_{-\rho} \mu_{\rho}\right) \subset \mathcal{L}_{\text {g.f. }} .
$$

Here $\vec{\rho}$ is a root of the Lie algebra. The first terms comes from $\operatorname{tr} b^{2}$ and the second term comes from $\operatorname{tr} \widetilde{c}\left[\Lambda_{0}, \widetilde{c}\right]$. Integrating over this mode and then calculating its variation with respect to $\alpha$ gives rise $\mathrm{BT}=1$. Since this contribution is a zero mode contribution and we are computing determinant over non zero mode, we subtract 1 from (3.67).

To treat the zero mode $c=\vec{A}$, we need to use the method of generalized Green's function. In this method, the Green's function equation is modified by a zero mode projector. Because of the presence of the zero mode projector, the variation of the one loop determinant, after performing integration by parts, now gives boundary terms together with an extra bulk term proportional to number of zero modes which now arises from the 
zero mode projector. Preliminary results from following this method results in extra -1 for (3.67). ${ }^{9}$ Thus taking into account all zero mode we get $\mathrm{BT}=0$ for the case $n=0=p$.

Thus, collecting all the above results we find that for $L^{2}>\frac{3}{4}$, the variation of the one loop determinant (2.32) is (for a general compact gauge group)

$$
\delta_{\vec{\alpha}} \ln Z=-\frac{i}{2} \sum_{n \neq 0} \frac{\vec{\rho}}{n-i \rho \cdot \alpha}-\frac{i}{2} \sum_{p \neq 0} \frac{\vec{\rho}}{\frac{p}{L}-i \rho \cdot \alpha} .
$$

Integrating with respect to $\alpha$, we obtain

$$
Z_{1-\mathrm{loop}}(\alpha)=\prod_{\rho} \sqrt{\prod_{n \neq 0}(n-i \rho \cdot \alpha) \prod_{p \neq 0}\left(\frac{p}{L}-i \rho \cdot \alpha\right)} .
$$

which is the result obtained in [17]. Thus, the partition function of a Chern Simons theory with level $k$ and the gauge group $G$ of rank $r$ is

$$
Z=\int_{\mathbb{R}^{r}} d \alpha \exp \left(-\pi i L k \operatorname{Tr} \alpha^{2}\right) \prod_{\rho>0} \sinh (\pi \rho \cdot \alpha) \sinh (\pi L \rho \cdot \alpha) .
$$

In the above, the integration variable $\alpha$ is valued in the Cartan of the Lie algebra of the gauge group $G$. Furthermore, we have also included the contribution of the Vandermonde determinant (it is the Jacobian coming from rotating any constant Lie algebra element to an element in the Cartan) to convert the infinite product (3.72) to the product of hyperbolic function.

If we also include matter field which consist of $N_{f}$ chiral multiplets transforming in some representation $\mathcal{R}_{i}$, where $i=1, \ldots, N_{f}$, of the gauge group, then the partition function of a Chern Simons theory matter theory is given by

$$
Z=\int_{\mathbb{R}^{r}} d \alpha \exp \left(-\pi i L k \operatorname{Tr} \alpha^{2}\right) \prod_{\rho>0} \sinh (\pi \rho \cdot \alpha) \sinh (\pi L \rho \cdot \alpha) \prod_{i=1}^{N_{f}} \prod_{\rho} Z_{\text {matter }}^{1 \text { llop }}\left(\mathcal{R}_{i} ; \alpha\right),
$$

where $Z_{\text {matter }}^{1 \text {-loop }}(\mathcal{R} ; \alpha)$ is the one loop determinant of the chiral multiplet in the representation $\mathcal{R}$. It was demonstrated in [18] using the Greens function method, to compute the one loop determinant $Z_{\text {matter }}^{1 \text {-loo }}(\mathcal{R} ; \alpha)$, that arises in Localization depends on the choice of $Q$-exact action. In particular, this difference arose for the modes in the interval $\frac{\Delta-1}{2 L}<n<\frac{\Delta}{2 L}$.

\section{Level-rank duality on $A d S_{2} \times S^{1}$}

In this section, we will discuss one of the implications of the result obtained in the last section for Chern Simons matter theory. We find that for the cases when there are no bulk terms in the partition function i.e. when the normalizable boundary conditions are consistent with supersymmetry (which is the case when $L^{2}>\frac{3}{4}$, and there are no integers

\footnotetext{
${ }^{9}$ The details of the treatment of the zero modes using the generalized Green's function as well as repeating the analysis in the standard covariant gauge as opposed to the gauge adopted in this work are presently in progress and will be presented in a future work.
} 
in the interval $\frac{\Delta-1}{2 L}<n<\frac{\Delta}{2 L}$ ), the partition function respects 3 dimension level-rank duality. We will consider here the example of $\mathrm{U}(N)$ Chern Simons theory coupled to $N_{f}$ hypermultiplets in fundamental (i.e. $N_{f}$ chiral in fundamental and $N_{f}$ chiral multiplet in anti fundamental) with R-charge $\Delta$. In this case the statement of level-rank duality is

$$
N_{f} \text { hypermultiplet coupled to } \mathrm{U}\left(N_{c}\right)_{k} \Longleftrightarrow N_{f} \text { hypermultiplet coupled to } \mathrm{U}\left(|k|+N_{f}-N_{c}\right)_{-k}
$$

We will find that this duality also holds true for $\mathrm{U}(N)$ Chern Simons theory coupled to $N_{f}$ hypermultiplets in fundamental on $A d S_{2} \times S^{1}$.

Without loss of generality we will assume that the ratio of the size $L=1$. However, one can generalize the discussion below for any value of $L$ such that $L^{2}>\frac{3}{4}$. Also for the presentation, we will also consider three different cases for which there are no integer in the interval $\frac{\Delta-1}{2 L}<n<\frac{\Delta}{2 L}$ : 1) with no matter fields $\left(N_{f}=0\right)$, 2) $N_{f}$ hypermultiplets in fundamental with R-charge $\Delta=0$, and 3) $N_{f}$ hypermultiplets in fundamental with R-charge $\Delta=1$.

Case: $\boldsymbol{N}_{\boldsymbol{f}}=\mathbf{0}$. In this case the partition function (3.74) reduces to the partition function of a pure Chern Simons theory which is

$$
Z=\int_{\mathbb{R}^{r}} d \alpha \exp \left(-\pi i k \operatorname{Tr} \alpha^{2}\right) \prod_{\rho>0} \sinh ^{2}(\pi \rho \cdot \alpha) .
$$

This partition function is exactly same as the partition function of $\mathrm{U}(N)_{k}$ Chern Simons theory on $\mathrm{S}^{3}$.

Case: $\boldsymbol{N}_{\boldsymbol{f}}$ hypermultiplets with $\boldsymbol{\Delta}=\mathbf{0}$. For a chiral multiplet with R-charge $\Delta=0$, the one loop contribution to the partition function (3.74) is given by

$$
\ln Z_{\text {matter }}^{1 \text {-loop }}=\sum_{p>0, n \geq 0} \ln (p+n+i \rho(\alpha))-\sum_{p \leq 0, n<0} \ln (-p-n-i \rho(\alpha)) .
$$

Thus for a given hypermultiplet with R-charge $\Delta=0$, the one loop contribution to the partition function is

$$
\begin{aligned}
\ln Z_{\text {hyper-matter }}^{\text {-loop }}= & \sum_{p>0, n \geq 0} \ln (p+n+i \rho(\alpha))-\sum_{p \leq 0, n<0} \ln (-p-n-i \rho(\alpha)) \\
& +\sum_{p>0, n \geq 0} \ln (p+n-i \rho(\alpha))-\sum_{p \leq 0, n<0} \ln (-p-n+i \rho(\alpha))=0 .
\end{aligned}
$$

Therefore, in this case there are no contribution to the partition function from the fields in the matter sector. Thus the partition function of $\mathrm{U}(N)_{k}$ Chern Simons theory coupled to $N_{f}$ hypermultiplet with R-charge $\Delta=0$ is equal to the partition function of $\mathrm{U}(N)_{k}$ Chern Simons theory. Note that this is same as the partition function of $\mathrm{U}(N)_{k}$ Chern Simons theory coupled to $N_{f}$ hypermultiplet on $\mathrm{S}^{3}$ but with R-charge $\Delta=1$. 
Case: $\boldsymbol{N}_{\boldsymbol{f}}$ hypermultiplets with $\boldsymbol{\Delta}=1$. For a chiral multiplet with R-charge $\Delta=1$, the one loop contribution to the partition function (3.74) is given by

$$
\ln Z_{\text {matter }}^{1 \text {-loop }}=\sum_{p>0, n \geq 1} \ln \left(p+n+i \rho(\alpha)-\frac{1}{2}\right)-\sum_{p \leq 0, n \leq 0} \ln \left(-p-n-i \rho(\alpha)+\frac{1}{2}\right) .
$$

Thus for a given hypermultiplet with R-charge $\Delta=1$, the one loop contribution to the partition function is

$$
Z_{\text {hyper-matter }}^{1 \text {-loop }}=\frac{\prod_{r=1}\left(r+i \rho(\alpha)+\frac{1}{2}\right)^{r}\left(r-i \rho(\alpha)+\frac{1}{2}\right)^{r}}{\prod_{r=1}\left(r-i \rho(\alpha)-\frac{1}{2}\right)^{r}\left(r+i \rho(\alpha)-\frac{1}{2}\right)^{r}}=\frac{1}{2 \cosh \pi \rho(\alpha)} .
$$

Therefore, the partition function of $\mathrm{U}(N)_{k}$ Chern Simons matter theory coupled to $N_{f}$ fundamental hypermultiplet with R-charge $\Delta=1$ is

$$
Z=\int_{\mathbb{R}^{r}} d \alpha \exp \left(-\pi i L k \operatorname{Tr} \alpha^{2}\right) \prod_{\rho>0} \sinh (\pi \rho \cdot \alpha) \sinh (\pi L \rho \cdot \alpha) \prod_{\rho}\left(\frac{1}{2 \cosh \pi \rho(\alpha)}\right)^{N_{f}}
$$

The above is the partition function of $\mathrm{U}(N)_{k}$ Chern Simons matter theory coupled to $N_{f}$ fundamental hypermultiplet on $\mathrm{S}^{3}$ with R-charge $\Delta=\frac{1}{2}$. It is known that this partition function respects the duality (4.1).

\section{Conclusions}

In this paper we have developed the method of Greens function introduced in [18] to evaluate one loop determinants that occur in localization of supersymmetric field theories on AdS spaces. The method requires the theory to have at least $\mathcal{N}=2$ supersymmetry in the respective space time dimensions. Boundary conditions of all fields play a crucial role in the application of localization in non-compact spaces. Normalizable boundary conditions are required for the definition of the path integral, it is only when normalizable boundary conditions are consistent with supersymmetric boundary conditions that the method of localization can be applied. We have introduced a general set of assumptions on the second order operators that occur in the evaluation of the one loop determinants that hold for theories with at least $\mathcal{N}=2$ supersymmetry. Under these assumptions we have constructed the Greens function and shown that the variation of the one loop determinant about the localizing background reduces to a total derivative. This is our first main result of the paper. This implies that the variation receives contributions only from asymptotic infinity and at the origin of AdS. Then from studying the asymptotics of the Greens function and the second order operators we show that the variation of the one loop determinant is given by an integer times the variation of $\frac{1}{2} \ln Q^{2}$. This is the second main result of our paper.

We then examine $\mathcal{N}=2$ Chern-Simons theory coupled to chiral multiplets on $A d S_{2} \times S^{1}$ and show how the general set of assumptions we introduced hold for this case. We use our results to conclude that $\mathrm{U}\left(N_{c}\right)$ Chern-Simons theory at level $k$ coupled to $N_{f}$ chiral multiplets and $N_{f}$ anti-chiral multiplets in the fundamental obeys level-rank duality on $A d S_{2} \times S^{1}$. 
As we have emphasised, the Greens function method is general as is applicable for other situations. We believe that the method is applicable to evaluate one loop determinants that arise in localization of supersymmetric theories on $A d S_{n} \times S^{m}$ with at least $\mathcal{N}=2$ supersymmetry. One such case is that of $\mathcal{N}=2$ theories on $A d S_{2} \times S^{2}$ with matter. We hope to report results related to this in the near future. Localization of supersymmetric field theories on $A d S_{2} \times S^{2}$ are relevant to evaluate quantum corrections to black hole entropy.

Another direction to explore will be localization of 2-dimensional theories on $A d S_{2}$. In particular it will be interesting to see if the duality between the Coulomb and the Higgs branch seen for $\mathcal{N}=(2,2)$ theories on the sphere $S^{2}$ by [21] also hold for the case of the theory on $A d S_{2}$.

The general method we have introduced can be further refined. The 8 assumptions presented in section 2 were obtained by a detailed study of the Greens function approach and extracting general properties. These assumptions enabled us to show that the variation of the one loop determinant reduces to a total derivative. We then introduced 3 assumptions in section 2.3. These set of assumptions enabled us to show that the variation of the one loop determinant is an integer times the variation of $\frac{1}{2} \ln Q^{2}$. Our preliminary investigations indicate that all these assumptions can be shown to hold true from the supersymmetry of the localizing Lagrangians. In fact we have seen that they also hold for $\mathcal{N}=2$ theories with matter on $A d S_{2} \times S^{2}$ [20]. It will be interesting to show that these assumptions follow as a natural consequence of supersymmetry.

Finally, we have seen that the Greens function method shows that variation of the one loop determinant is given by integer times the variation of $\frac{1}{2} \ln Q^{2}$. We again emphasise that this result is only when normalizable boundary conditions are compatible with supersymmetry. The integer is given by the index of the operator $C$ restricted to a $k$-dimensional vector space. It will be interesting to investigate if this result can be connected with the technique of applying the fixed point evaluation of one loop determinants that arise in localization as recently applied in [13, 16, 22-24].

\section{Acknowledgments}

We thank Sameer Murthy for useful conversations. The work of RG is supported by the ERC Consolidator Grant N. 681908, "Quantum black holes: A macroscopic window into the microstructure of gravity".

\section{A Supersymmetry of the vector multiplet}

Vector multiplet in $\mathcal{N}=2$ theory in Lorentzian signature contains a real scalar $\sigma$, gauge field $a_{\mu}$, an auxiliary real field $G$ and 2 component Weyl fermions $\lambda$ and $\tilde{\lambda}$. In order to compute partition function we need to analytically continue to Euclidean space. We choose the analytic continuation where the scalar field $\sigma$ and the auxiliary field $H$ are purely imaginary, the gauge field $a_{\mu}$ is real and the spinors $\lambda$ and $\tilde{\lambda}$ are two independent complex spinor. The Euclidean supersymmetry transformation of the fields in the vector 
multiplet is given by

$$
\begin{aligned}
Q_{s} \lambda & =-\frac{i}{4} \epsilon H-\frac{i}{2} \epsilon^{\mu \nu \rho} \gamma_{\rho} F_{\mu \nu} \epsilon-i \gamma^{\mu} \epsilon\left(i D_{\mu} \sigma-V_{\mu} \sigma\right), \\
Q_{s} \tilde{\lambda} & =\frac{i}{4} \tilde{\epsilon} H-\frac{i}{2} \epsilon^{\mu \nu \rho} \gamma_{\rho} F_{\mu \nu} \tilde{\epsilon}+i \gamma^{\mu} \tilde{\epsilon}\left(i D_{\mu} \sigma+V_{\mu} \sigma\right), \\
Q_{s} a_{\mu} & =\frac{1}{2}\left(\epsilon \gamma_{\mu} \tilde{\lambda}+\tilde{\epsilon} \gamma_{\mu} \lambda\right), \\
Q_{s} \sigma & =\frac{1}{2}(-\epsilon \tilde{\lambda}+\tilde{\epsilon} \lambda) \\
Q_{s} H & =-2 i\left[D_{\mu}\left(\epsilon \gamma^{\mu} \tilde{\lambda}-\tilde{\epsilon} \gamma^{\mu} \lambda\right)-i[\sigma, \epsilon \tilde{\lambda}+\tilde{\epsilon} \lambda]-i V_{\mu}\left(\epsilon \gamma^{\mu} \tilde{\lambda}+\tilde{\epsilon} \gamma^{\mu} \lambda\right)\right] .
\end{aligned}
$$

The square of the susy transformations on vector multiplet fields are given by

$$
\begin{aligned}
Q_{s}^{2} \lambda & =\mathcal{L}_{K} \lambda+i[\Lambda, \lambda]-\frac{1}{2 L} \lambda, \\
Q_{s}^{2} \tilde{\lambda} & =\mathcal{L}_{K} \tilde{\lambda}+i[\Lambda, \tilde{\lambda}]+\frac{1}{2 L} \tilde{\lambda}, \\
Q_{s}^{2} a_{\mu} & =\mathcal{L}_{K} a_{\mu}+D_{\mu} \Lambda, \\
Q_{s}^{2} \sigma & =\mathcal{L}_{K} \sigma-i K^{\mu}\left[a_{\mu}, \sigma\right], \\
Q_{s}^{2} H & =\mathcal{L}_{K} H+i[\Lambda, H] .
\end{aligned}
$$

Here $\Lambda=\tilde{\epsilon} \epsilon \sigma-K^{\rho} a_{\rho}$.

Using the above supersymmetry transformations we also note that $Q_{s} \Lambda=0$.

Therefore, the algebra of supersymmetry transformation is given by

$$
Q_{s}^{2}=\mathcal{L}_{K}+\delta_{\Lambda}^{\text {gauge transf }}+\delta_{\frac{1}{2 L}}^{R-\text { symm }} .
$$

\section{B Equations of motions}

The equations of motion for the vector field and scalar field can be written as

$$
M_{2} \partial_{r}^{2} E^{+}(r)+M_{1} \partial_{r} E^{+}(r)+M_{0} E^{+}(r)=0
$$

Here $M_{2,1,0}$ are $4 \times 4$ matrices which are functions of coordinate $r$, and

$$
E^{+}(r)=\left(\begin{array}{c}
a_{t}^{+}(r) \\
a_{r}^{+}(r) \\
a_{\theta}^{+}(r) \\
\sigma^{+}(r)
\end{array}\right)
$$


with components

$$
M_{2}=\left(\begin{array}{cccc}
-\frac{\sinh r}{2} & 0 & 0 & 0 \\
0 & \frac{\sinh r}{4 L^{2} \xi} & 0 & 0 \\
0 & 0 & -\frac{1}{2 L^{2} \sinh r} & 0 \\
0 & 0 & 0 & \frac{\sinh r}{2}
\end{array}\right)
$$

$$
M_{1,11}=-\frac{\cosh r}{2}, \quad M_{1,12}=-\frac{1}{4 \xi}\left(n(1+2 \xi) \cosh ^{2} r-2 \xi \alpha\right) \operatorname{sech} r \tanh r
$$$$
M_{1,13}=0, \quad M_{1,14}=0
$$$$
M_{1,21}=\frac{1}{4 \xi}\left(n(1+2 \xi) \cosh ^{2} r-2 \xi \alpha\right) \operatorname{sech} r \tanh r, \quad M_{1,22}=\frac{\cosh r}{4 L^{2} \xi}
$$$$
M_{1,23}=\frac{1}{4 L^{2} \xi \sinh r \cosh ^{2} r}\left(p(1+2 \xi) \cosh ^{2} r-2 L \xi \sinh ^{2} r\right), \quad M_{1,24}=0
$$$$
M_{1,31}=0, \quad M_{1,32}=-\frac{1}{4 L^{2} \xi \sinh r \cosh ^{2} r}\left(p(1+2 \xi) \cosh ^{2} r-2 L \xi \sinh ^{2} r\right)
$$$$
M_{1,33}=\frac{\cosh r}{2 L^{2} \sinh ^{2} r}, \quad M_{1,34}=0, \quad M_{1,41}=0, \quad M_{1,42}=0, \quad M_{1,43}=0
$$$$
M_{1,44}=\frac{\cosh r}{2}
$$

$$
M_{0,11}=\frac{p^{2}}{2 \sinh r}+\frac{L^{2}}{2 \xi}\left(-n^{2} \sinh r+2 \xi(2 n-\alpha) \alpha \operatorname{sech} r \tanh r\right)
$$$$
M_{0,12}=\alpha \operatorname{sech}^{3} r+\frac{1}{4 \xi}(n(1-2 \xi) \cosh r-2(n+\xi \alpha) \operatorname{sech} r)
$$$$
M_{0,13}=\frac{1}{2}(L n-p) \alpha \operatorname{sech} r \tanh r-\frac{1}{4 \xi \sinh r}(p(n+2 n \xi-2 \alpha \xi)),
$$$$
M_{0,14}=0, \quad M_{0,21}=\frac{n \sinh r \tanh r}{2 \xi}
$$$$
M_{0,22}=\frac{1}{2}\left(n^{2} \sinh r-\alpha^{2} \operatorname{sech} r \tanh r\right)+\frac{1}{4 L^{2} \xi \sinh r}\left(-1+2 p^{2} \xi+2 \sinh ^{2} r \tanh ^{2} r\right)
$$$$
M_{0,23}=-\frac{p}{L^{2} \xi \sinh r \sinh 2 r}, \quad M_{0,24}=0
$$$$
M_{0,31}=\frac{1}{2}(L n-p) \alpha \operatorname{sech} r \tanh r-\frac{1}{4 \xi \sinh r}(p(n+2 n \xi-2 \alpha \xi))
$$$$
M_{0,32}=\frac{1}{4 L^{2} \xi}\left(p(-1+2 \xi) \frac{\cosh r}{\sinh ^{2} r}+2 \operatorname{sech} r\left(p-L \xi \alpha+2 L \xi \alpha \operatorname{sech}^{2} r\right)\right)
$$$$
M_{0,33}=\frac{1}{2 L \sinh r}\left(2 p \alpha+L\left(n^{2}-\alpha^{2}\right)\right)-\frac{p^{2}}{4 L^{2} \xi \sinh ^{3} r}+\frac{\xi}{2 L}(-2 p+L \alpha) \operatorname{sech} r \tanh r,
$$ 


$$
\begin{aligned}
& M_{0,34}=0, \quad M_{0,41}=0, \quad M_{0,42}=0, \quad M_{0,43}=0 \\
& M_{0,44}=-\frac{p^{2}}{2 \sinh r}-\frac{1}{2} L^{2} n^{2} \sinh r+\operatorname{sech} r \tanh r .
\end{aligned}
$$

In the case of fermions, we get

$M_{2 f, 1 i}=\delta_{i 4} \frac{1}{2} \operatorname{sech} r \tanh r, \quad M_{2 f, 2 i}=0, \quad M_{2 f, 3 i}=\delta_{i 4} \frac{1}{2 L} \operatorname{sech} r \tanh r$

$M_{2 f, 5 i}=0, \quad M_{2 f, 6 i}=0$,

$M_{2 f, 41}=-\frac{1}{2} \operatorname{sech} r \tanh r, \quad M_{2 f, 42}=0, \quad M_{2 f, 43}=-\frac{1}{2 L} \operatorname{sech} r \tanh r$,

$M_{2 f, 44}=\frac{i}{2 L}(p+L(n-\alpha)) \operatorname{sech} r \tanh r, \quad M_{2 f, 45}=0, \quad M_{2 f, 46}=0$

$M_{1 f, 11}=0, \quad M_{1 f, 12}=-\frac{i}{2} \operatorname{sech} r \tanh r, \quad M_{1 f, 13}=0, \quad M_{1 f, 14}=\frac{1}{4}(3-\cosh 2 r) \operatorname{sech}^{3} r$

$M_{1 f, 15}=0, \quad M_{1 f, 16}=-\frac{1}{2} L \tanh ^{2} r, \quad M_{1 f, 21}=\frac{i}{2} \operatorname{sech} r \tanh r, \quad M_{1 f, 22}=0$

$M_{1 f, 23}=\frac{i}{2 L} \operatorname{sech} r \tanh r, \quad M_{1 f, 24}=0, \quad M_{1 f, 25}=-\frac{i}{2} \sinh r, \quad M_{1 f, 26}=0$,

$M_{1 f, 31}=0, \quad M_{1 f, 32}=-\frac{i}{2 L} \operatorname{sech} r \tanh r, \quad M_{1 f, 33}=0, \quad M_{1 f, 34}=\frac{1}{4 L}(3-\cosh 2 r) \operatorname{sech}^{3} r$

$M_{1 f, 35}=0, \quad M_{1 f, 36}=\frac{1}{2} \operatorname{sech}^{2} r, \quad M_{1 f, 41}=\frac{1}{4 \cosh ^{3} r}(-3+\cosh 2 r), \quad M_{1 f, 42}=0$

$M_{1 f, 43}=\frac{1}{4 L \cosh ^{3} r}(-3+\cosh 2 r), \quad M_{1 f, 44}=-\frac{i(p+L(n-\alpha))}{4 L \cosh ^{3} r}(-3+\cosh 2 r)$

$M_{1 f, 45}=0, \quad M_{1 f, 46}=0, \quad M_{1 f, 51}=0, \quad M_{1 f, 52}=\frac{i}{2} \sinh r, \quad M_{1 f, 53}=0, \quad M_{1 f, 54}=0$,

$M_{1 f, 55}=0, \quad M_{1 f, 56}=0, \quad M_{1 f, 61}=-\frac{L}{2} \tanh ^{2} r, \quad M_{1 f, 62}=0, \quad M_{1 f, 63}=\frac{1}{2} \operatorname{sech}^{2} r$

$M_{1 f, 64}=0, \quad M_{1 f, 65}=0, \quad M_{1 f, 66}=0$

$M_{0 f, 11}=-\frac{i L}{2}(-p+L(n-\alpha)) \operatorname{sech} r \tanh r, \quad M_{0 f, 12}=\frac{i}{4 \cosh ^{3} r}(-3+\cosh 2 r)$

$M_{0 f, 13}=-\frac{i}{2} \operatorname{sech}^{2} r\left(L n \sinh r+\frac{p}{\sinh r}\right), \quad M_{0 f, 14}=-\frac{1}{2} \operatorname{sech}^{2} r\left(L^{2} n^{2} \sinh r+\frac{p^{2}}{\sinh r}\right)$

$M_{0 f, 15}=\frac{i}{2} L^{2} n \sinh r, \quad M_{0 f, 16}=-L \operatorname{sech}^{2} r \tanh r, \quad M_{0 f, 21}=0$

$M_{0 f, 22}=\frac{i}{2 L}(p+L(n+\alpha)) \operatorname{sech} r \tanh r, \quad M_{0 f, 23}=0, \quad M_{0 f, 24}=0, \quad M_{0 f, 25}=-i \sinh r \tanh r$,

$M_{0 f, 26}=\frac{1}{2}\left(\operatorname{Ln} \sinh ^{2} r-p\right) \operatorname{sech}^{2} r, \quad M_{0 f, 31}=-\frac{i}{2} \operatorname{sech}^{2} r\left(\operatorname{Ln} \sinh r+\frac{p}{\sinh r}\right)$,

$M_{0 f, 32}=\frac{i}{4 L \cosh ^{3} r}(-3+\cosh 2 r), \quad M_{0 f, 33}=\frac{i}{2 L \sinh r \cosh ^{2} r}(-p+L(n+\alpha))$,

$M_{0 f, 34}=-\frac{1}{2 L} \operatorname{sech}^{2} r\left(L^{2} n^{2} \sinh r+\frac{p^{2}}{\sinh r}\right), \quad M_{0 f, 35}=\frac{i p}{2 \sinh r}, \quad M_{0 f, 36}=-\operatorname{sech}^{2} r \tanh r$

$M_{0 f, 41}=\frac{1}{2} \operatorname{sech}^{2} r\left(L^{2} n^{2} \sinh r+\frac{p^{2}}{\sinh r}\right), \quad M_{0 f, 42}=0, \quad M_{0 f, 43}=\frac{1}{2 L} \operatorname{sech}^{2} r\left(L^{2} n^{2} \sinh r+\frac{p^{2}}{\sinh r}\right)$

$M_{0 f, 44}=-\frac{i(p+L(n-\alpha))}{2 L \sinh r \cosh ^{2} r}\left(L^{2} n^{2} \sinh ^{2} r+p^{2}\right), \quad M_{0 f, 45}=0, \quad M_{0 f, 46}=0$

$M_{0 f, 51}=\frac{i}{2} L^{2} n \sinh r, \quad M_{0 f, 52}=-\frac{i}{4 \cosh r}(-3+\cosh 2 r), \quad M_{0 f, 53}=\frac{i p}{2 \sinh r}, \quad M_{0 f, 54}=0$, 


$$
\begin{aligned}
& M_{0 f, 55}=i L \xi(p+L(n-\alpha)) \sinh r, \quad M_{0 f, 56}=0, \quad M_{0 f, 61}=0, \quad M_{0 f, 62}=-\frac{1}{2}\left(L n \sinh ^{2} r-p\right) \operatorname{sech}^{2} r \\
& M_{0 f, 63}=0, \quad M_{0 f, 64}=0, \quad M_{0 f, 65}=0, \quad M_{0 f, 66}=-\frac{i L}{2}(p+L(n-\alpha)) \operatorname{sech} r \tanh r .
\end{aligned}
$$

\section{On gauge fixing conditions}

Here we justify the choice of the gauge fixing condition (3.10). In particular, we will show that the one loop result obtained for the abelian gauge theory in [17] using the covariant gauge also holds true for the gauge fixing condition chosen in this paper. In fact, it works for the general gauge fixing condition

$$
G(a)=\cosh ^{\delta} r \nabla_{\mu}\left(\frac{1}{\cosh ^{\delta} r} a^{\mu}\right)=\cosh ^{\delta} r \nabla_{\hat{\mu}}\left(\frac{1}{\cosh ^{\delta} r} a^{\hat{\mu}}\right)+\partial_{t} a_{t} .
$$

where $\hat{\mu}$ is 2-dim AdS indices. The integral over ghost gives the Jacobian $J$ which is defined through the functional integral as:

$$
\int \mathcal{D} \lambda J \delta(M \lambda)=1
$$

where $M$ is obtained by infinitesimal gauge transformation $a \rightarrow a+d \lambda$ on $G$ :

$$
M=\cosh ^{\delta} r \nabla_{\hat{\mu}}\left(\frac{g^{\hat{\mu} \hat{\nu}}}{\cosh ^{\delta} r} \nabla_{\hat{\nu}}\right)+\partial_{t}^{2}
$$

and $\lambda$ is in the space of all allowed gauge transformations.

Now, the allowed gauge transformations are defined as the ones that preserve the square integrability of gauge fields $\left(a, a_{t}\right)$ : for $t$-dependent part of the gauge transformation parameter $\lambda(t, r, \theta)=\sum_{n \neq 0} \lambda_{n}(r, \theta) e^{\text {int }}$, it requires that

$$
e^{r / 2} \lambda_{n}(r, \theta) \rightarrow 0, \quad \text { for } r \rightarrow \infty
$$

The space of such gauge transformation we denote by $\mathcal{H}$. However, for $t$-independent part of the gauge transformation parameter $\lambda_{0}(r, \theta)$, the condition on the normalizability of the gauge field requires that

$$
\lambda_{0}(r, \theta) \sim \lambda_{0}^{(0)}(\theta)+e^{-\beta r / 2} \lambda_{0}^{(\beta)}(\theta)+\ldots, \quad \text { for } \beta>1
$$

The space of such gauge transformation we denote by $\mathcal{H}_{0}$. We note that the operator $M$ is not self-adjoint for $\delta \neq 0$. However, it has the following properties:

1) We first note that the differential operator $M$ does not have zero modes for $\delta>1$. We find that the solution of $M f(r)=0$ has following large $r$ asymptotic

$$
f(r)= \begin{cases}e^{-\frac{r}{2}\left(1-\delta-\sqrt{4 L^{2} n^{2}+(1-\delta)^{2}}\right)}, & \text { for } n \neq 0 \\ e^{r(\delta-1)}, & \text { for } n=0, \delta>1, \\ \mathcal{O}(1), & \text { for } n=0,0 \leq \delta \leq 1 .\end{cases}
$$


From the above we see that for $n \neq 0$, there are no zero mode. For $n=0$, we have zero modes for $\delta \leq 1$. Thus for $\delta=2$, which is our choice of gauge, we do not have zero modes and therefore, the gauge choice completely fixes the gauge. For $\delta=0$, which corresponds to covariant gauge fixing, there are an infinite number of zero modes [25]. Now, we will solve the differential equation for the adjoint operator, $M^{\dagger} \bar{f}(r)=0$. Solutions have following large $r$ asymptotic behaviour

$$
\bar{f}(r)= \begin{cases}e^{-\frac{r}{2}\left(1+\delta-\sqrt{4 L^{2} n^{2}+(1-\delta)^{2}}\right)}, & \text { for } n \neq 0 \\ e^{-r}, & \text { for } n=0, \alpha>1, \\ e^{-\delta r}, & \text { for } n=0,0 \leq \delta<1 \\ \Gamma(0), & \text { for } n=0, \delta=1 .\end{cases}
$$

So, we see that for $n \neq 0$, there are no zero modes if $L^{2} n^{2} \geq \frac{2 \delta-1}{4}$. In particular, for $\delta=2$, which is our gauge fixing, and $L^{2}>\frac{3}{4}$, which is supersymmetric case, there are no zero modes. For $0 \leq \delta \leq 1$, we do not have zero modes.

2) For $n \neq 0, M \mathcal{H}$ spans all of $\mathcal{H}$. The argument is as follows: if we assume that there must exist some function say $f^{\prime}$ such that it is orthogonal to $M f$ for all $f \in \mathcal{H}$, i.e. $\int d^{2} x f^{\prime} M f=0$ for all $f \in \mathcal{H}$, then integrating by part, we get $M^{\dagger} f^{\prime}=0$. In this computation we obtain boundary terms which vanish because both $f, f^{\prime} \in \mathcal{H}$. But as we have shown before that for $n \neq 0$, kernel of $M^{\dagger}$ in $\mathcal{H}$ is empty. This proves that $M \mathcal{H}$ spans all of $\mathcal{H}$.

3) For $n=0, \lambda \in \mathcal{H}_{0}$. Now one can see that $M \mathcal{H}_{0}$ is contained in $\mathcal{H}$. Furthermore, $M$ has no kernel in $\mathcal{H}_{0}$ for $\delta \geq 1$ while for $\delta<1$ it has a kernel with the zero mode going to order one asymptotically. This means that for $\delta<1$ the large gauge transformations are not fixed.

We will now perform the path integral for each Fourier mode $n$ along $t$. For $n \neq 0$ we can solve $G(a)=0$ for $a_{t}$ as

$$
a_{t}^{(n)}=\frac{i}{n} \cosh ^{\delta} r \nabla_{\hat{\mu}}\left(\frac{g^{\hat{\mu} \hat{\nu}}}{\cosh ^{\delta} r} a_{\hat{\nu}}^{(n)}\right)
$$

Integrating $a_{t}$ for $n \neq 0$, one also gets $\prod_{n \neq 0} \frac{1}{n}$. For $n=0$, we are left with the Gauge fixing condition $\cosh ^{\delta} r \nabla_{\hat{\mu}}\left(\frac{g^{\hat{\mu} \hat{\nu}}}{\cosh ^{\delta} r} a_{\hat{\nu}}^{(0)}\right)=0$.

For the other component of the gauge field, we use the 2-dim hodge decomposition

$$
a_{\hat{\mu}}^{(n)}=\partial_{\hat{\mu}} f_{n}+\epsilon_{\hat{\mu} \hat{\nu}} \partial^{\hat{\nu}} f_{n}^{\prime}, \quad \text { where } \epsilon_{r \theta}=\sqrt{\hat{g}}=L^{2} \sinh r .
$$

Here $f^{\prime} \in \mathcal{H}$ while $f \in \mathcal{H}_{0}$. Furthermore, we split $f$ as $\hat{f}+g$ where now $\hat{f} \in \mathcal{H}$ and $g \in \mathcal{H}_{0} / \mathcal{H}$, i.e. $g$ can go as $\mathcal{O}(1)$ near $r \rightarrow \infty$. Moreover, we demand that $g$ is orthogonal to all the normalizable fns $\hat{f}$ (and $f^{\prime}$ ) with respect to the inner product $\int d^{2} x g^{\hat{\mu} \hat{\nu}} \partial_{\hat{\mu}} g \partial_{\hat{\nu}} \hat{f}=0$. This can be satisfied by taking $g$ to be solution of AdS laplacian (i.e. discreet modes). 
More explicitly a normalized basis with respect to the above norm for these solutions is $g=\frac{1}{\sqrt{2 \pi|\ell|}} g_{n, p} \tanh ^{|\ell|}\left(\frac{r}{2}\right) e^{i \ell \theta} e^{\text {int }}$, where $\ell= \pm 1, \pm 2, \ldots$.

The Chern Simons action upto total derivative terms becomes

$$
\begin{aligned}
& \frac{\kappa}{2} \int d^{2} x \sum_{n>0}\left[\frac{2 i}{n}\left(\left(M \hat{f}_{n}\right) \square f_{-n}^{\prime}-\left(M \hat{f}_{-n}\right) \square f_{n}^{\prime}\right)+\ldots\right]+\frac{\kappa}{2} \int d^{2} x a_{t}^{(0)} \square f_{0}^{\prime} \\
& +\kappa \sum_{n, \ell>0} n\left(g_{n,-\ell} g_{-n, \ell}-g_{n, \ell} g_{-n,-\ell}\right) .
\end{aligned}
$$

Here $\square$ is a $2 \mathrm{AdS}_{2}$ Laplacian and dots include terms that do not involve $\hat{f}_{n}$ and $\hat{f}_{-n}$ and vanish when $f_{n}^{\prime}$ and $f_{-n}^{\prime}$ vanish. Next, we want to change the variables from $f \rightarrow \widetilde{f}=M \hat{f}$. This can be done in the functional integral by inserting

$$
\int \prod_{n \neq 0} \mathcal{D} \tilde{f}_{n} \mathcal{D} \tilde{f}_{-n} \delta\left(\tilde{f}_{n}-M \hat{f}_{n}\right) \delta\left(\tilde{f}_{-n}-M \hat{f}_{-n}\right)=1,
$$

and integrate first $\hat{f}_{n}$ and $\hat{f}_{-n}$. Now, taking into account the Fadeev Popov determinant $J$ (for $n \neq 0$ ) satisfying (C.2) and noting the fact that $\hat{f}$ and $\lambda$ are in the same space of normalizable functions, the result of this integral together with $J$, is simply to replace $M \hat{f}_{n}$ and $M \hat{f}_{-n}$ by $\widetilde{f}_{n}$ and $\widetilde{f}_{-n}$, respectively in the action (C.11). From the argument in (2) above $\widetilde{f}$ spans all of $\mathcal{H}$. Now we can integrate $\widetilde{f}_{n}$ and $\widetilde{f}_{-n}$ and the result is $\prod_{n \neq 0} \delta\left(\frac{i \kappa}{n} \square f_{n}^{\prime}\right) \delta\left(\frac{-i \kappa}{n} \square f_{-n}^{\prime}\right)$. Similarly, integrating the auxiliary fields $a_{t}^{(0)}$ we get $\delta\left(i \kappa \square f_{0}^{\prime}\right)$. Since $f^{\prime} \in \mathcal{H}$ and $\square$ has no zero mode in $\mathcal{H}$, delta function enforces $f_{n}^{\prime}=0$ in the remaining part of the action i.e. the dots in (C.11) vanish. Finally integrating $f_{n}^{\prime}$ and $f_{-n}^{\prime}$ we get $\frac{1}{\operatorname{det} \kappa \square} \prod_{n \neq 0} \frac{1}{\operatorname{det}\left(\frac{\kappa}{n} \square\right)}$.

Now, we are still left with the integral over $f_{0}$ and $\delta\left(M f_{0}\right)$ in the integrand (coming from the gauge condition for $n=0$ ) where $f_{0} \in \mathcal{H}_{0}$ includes both square integrable as well as functions that go as order one at infinity. However, the contribution of this integral is exactly cancelled by the Fadeev Popov determinant $J$ for $n=0$. Finally, we perform the integral over $g_{n, \ell}$ and take the logarithm of the entire result. We get

$$
-\sum_{n \in \mathbb{Z}} \ln (k \square)-\sum_{n, \ell=1}^{\infty} \ln k^{2}=-\frac{1}{2} \ln k .
$$

In the above we have used zeta function regularization. This is the same result as obtained in [17] using the covariant gauge fixing condition.

Open Access. This article is distributed under the terms of the Creative Commons Attribution License (CC-BY 4.0), which permits any use, distribution and reproduction in any medium, provided the original author(s) and source are credited.

\section{References}

[1] V. Pestun et al., Localization techniques in quantum field theories, J. Phys. A 50 (2017) 440301 [arXiv:1608.02952] [InSPIRE].

[2] E. Witten, Two-dimensional gauge theories revisited, J. Geom. Phys. 9 (1992) 303 [hep-th/9204083] [INSPIRE]. 
[3] N.A. Nekrasov, Seiberg-Witten prepotential from instanton counting, in proceedings of the International Congress of Mathematicians (ICM 2002), Beijing, China, 20-28 August 2002, hep-th/0306211 [INSPIRE].

[4] N.A. Nekrasov and A. Okounkov, Seiberg-Witten theory and random partitions, Prog. Math. 244 (2006) 525 [hep-th/0306238] [InSPIRE].

[5] V. Pestun, Localization of gauge theory on a four-sphere and supersymmetric Wilson loops, Commun. Math. Phys. 313 (2012) 71 [arXiv:0712.2824] [INSPIRE].

[6] M. Mariño and P. Putrov, Exact Results in ABJM Theory from Topological Strings, JHEP 06 (2010) 011 [arXiv:0912.3074] [INSPIRE].

[7] F. Benini, K. Hristov and A. Zaffaroni, Exact microstate counting for dyonic black holes in $A d S_{4}$, Phys. Lett. B 771 (2017) 462 [arXiv:1608.07294] [InSPIRE].

[8] F. Benini, K. Hristov and A. Zaffaroni, Black hole microstates in AdS $S_{4}$ from supersymmetric localization, JHEP 05 (2016) 054 [arXiv:1511.04085] [INSPIRE].

[9] A. Dabholkar, J. Gomes and S. Murthy, Quantum black holes, localization and the topological string, JHEP 06 (2011) 019 [arXiv: 1012.0265] [INSPIRE].

[10] A. Dabholkar, J. Gomes and S. Murthy, Localization \& Exact Holography, JHEP 04 (2013) 062 [arXiv:1111.1161] [InSPIRE].

[11] R.K. Gupta and S. Murthy, All solutions of the localization equations for $N=2$ quantum black hole entropy, JHEP 02 (2013) 141 [arXiv:1208.6221] [INSPIRE].

[12] A. Dabholkar, J. Gomes and S. Murthy, Nonperturbative black hole entropy and Kloosterman sums, JHEP 03 (2015) 074 [arXiv: 1404.0033] [INSPIRE].

[13] S. Murthy and V. Reys, Functional determinants, index theorems and exact quantum black hole entropy, JHEP 12 (2015) 028 [arXiv: 1504.01400] [INSPIRE].

[14] R.K. Gupta, Y. Ito and I. Jeon, Supersymmetric Localization for BPS Black Hole Entropy: 1-loop Partition Function from Vector Multiplets, JHEP 11 (2015) 197 [arXiv:1504.01700] [INSPIRE].

[15] A. Dabholkar, N. Drukker and J. Gomes, Localization in supergravity and quantum $A d S_{4} / C F T_{3}$ holography, JHEP 10 (2014) 090 [arXiv:1406.0505] [INSPIRE].

[16] A. Cabo-Bizet, V.I. Giraldo-Rivera and L.A. Pando Zayas, Microstate counting of AdS 4 hyperbolic black hole entropy via the topologically twisted index, JHEP 08 (2017) 023 [arXiv: 1701.07893] [INSPIRE].

[17] J.R. David, E. Gava, R.K. Gupta and K. Narain, Localization on $A d S_{2} \times S^{1}$, JHEP 03 (2017) 050 [arXiv: 1609.07443] [INSPIRE].

[18] J.R. David, E. Gava, R.K. Gupta and K. Narain, Boundary conditions and localization on AdS. Part I, JHEP 09 (2018) 063 [arXiv: 1802.00427] [InSPIRE].

[19] A. Pittelli, Supersymmetric localization of refined chiral multiplets on topologically twisted $H^{2} \times S^{1}$, Phys. Lett. B 801 (2020) 135154 [arXiv:1812.11151] [INSPIRE].

[20] J.R. David, E. Gava, R.K. Gupta and K. Narain, Boundary conditions and localization on AdS. Part III, to appear.

[21] N. Doroud, J. Gomis, B. Le Floch and S. Lee, Exact Results in D $=2$ Supersymmetric Gauge Theories, JHEP 05 (2013) 093 [arXiv:1206.2606] [INSPIRE]. 
[22] B. Assel, D. Martelli, S. Murthy and D. Yokoyama, Localization of supersymmetric field theories on non-compact hyperbolic three-manifolds, JHEP 03 (2017) 095

[arXiv: 1609.08071] [INSPIRE].

[23] B. de Wit, S. Murthy and V. Reys, BRST quantization and equivariant cohomology: localization with asymptotic boundaries, JHEP 09 (2018) 084 [arXiv:1806.03690] [INSPIRE].

[24] I. Jeon and S. Murthy, Twisting and localization in supergravity: equivariant cohomology of BPS black holes, JHEP 03 (2019) 140 [arXiv: 1806.04479] [INSPIRE].

[25] S. Banerjee, R.K. Gupta and A. Sen, Logarithmic Corrections to Extremal Black Hole Entropy from Quantum Entropy Function, JHEP 03 (2011) 147 [arXiv:1005.3044] [INSPIRE]. 